\title{
The Existence of Chaos in Infinite Dimensional Non-Resonant Systems
}

\author{
Michal Fečkan and Joseph Gruendler \\ Communicated by Y. Charles Li, received July 21, 2008.
}

\begin{abstract}
This work is concerned with showing the existence of chaotic dynamics in the flow generated by an infinite system of strongly coupled ordinary differential equations with a finite dimensional hyperbolic part and an infinite dimensional center part. This theory can be applied to partial differential equations by using a Galerkin expansion which is illustrated by the problem of oscillations of a buckled elastic beam.
\end{abstract}

\section{Contents}

1. Introduction 185

2. The Abstract Problem 189

3. Chaos on the Hyperbolic Subspace 190

4. Chaos in the Full Equation 192

5. Applications: Vibrating Elastic Beams 193

$\begin{array}{ll}\text { Appendix A. Appendix } & 205\end{array}$

$\begin{array}{ll}\text { References } & 207\end{array}$

\section{Introduction}

To motivate the ideas of this work consider the partial differential equation

$$
\ddot{u}=-u^{\prime \prime \prime \prime}-P_{0} u^{\prime \prime}+\left[\int_{0}^{\pi} u^{\prime}(s)^{2} d s\right] u^{\prime \prime}-2 \mu_{2} \dot{u}+\mu_{1} \cos \omega_{0} t
$$

where $P_{0}, \mu_{1}, \mu_{2}, \omega_{0}$ are constants and $u$ is a real valued function of two variables $t \in \mathbb{R}, x \in[0, \pi]$, subject to the boundary conditions

$$
u(0, t)=u(\pi, t)=u^{\prime \prime}(0, t)=u^{\prime \prime}(\pi, t)=0 .
$$

1991 Mathematics Subject Classification. 34C37, 35B99, 74H65.

Key words and phrases. Differential equations, homoclinic solutions, bifurcations, center manifold, chaos.

Fečkan's work was supported by the Grant VEGA-SAV 2/7140/27.

(C)2008 International Press 


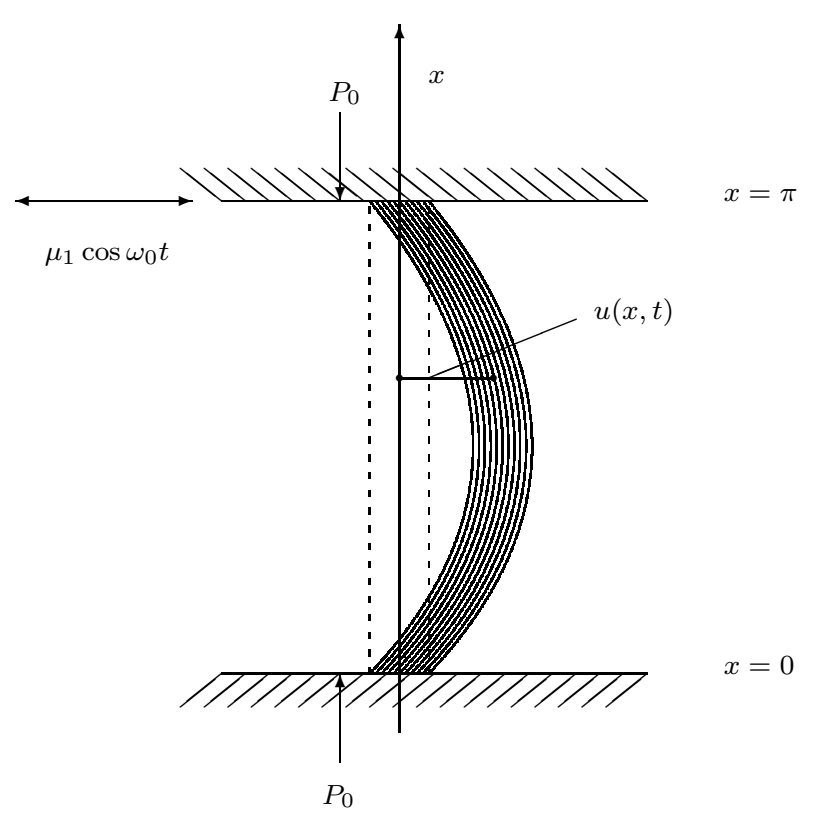

Figure 1. The forced buckled beam (1.1).

In (1.1), a superior dot denotes differentiation with respect to $t$ and prime differentiation with respect to $x$. This is a model for oscillations of an elastic beam with a compressive axial load $P_{0}$ (see Figure 1). When $P_{0}$ is sufficiently large, (1.1) can exhibit chaotic behavior. The first work on this was done by Holmes and Marsden [18]. Some more recent work on the full equation is by Rodrigues and Silveira [32], by Berti and Carminati [4] and by Battelli, Fečkan and Franca [2]. An undamped buckled beam is investigated by Yagasaki [41] to show Arnold diffusion type motions. We will discuss some of this in more detail when we return to this problem in Section 5.

In (1.1) substitute $u(x, t)=\sum_{k=1}^{\infty} u_{k}(t) \sin k x$, multiply by $\sin n x$ and integrate from 0 to $\pi$. This yields the infinite set of ordinary differential equations

$$
\begin{gathered}
\ddot{u}_{n}=n^{2}\left(P_{0}-n^{2}\right) u_{n}-\frac{\pi}{2} n^{2}\left[\sum_{k=1}^{\infty} k^{2} u_{k}^{2}\right] u_{n}-2 \mu_{2} \dot{u}_{n}+2 \mu_{1}\left[\frac{1-(-1)^{n}}{\pi n}\right] \cos \omega_{0} t \\
n=1,2, \ldots
\end{gathered}
$$

We see that the linear parts of these equations are uncoupled and the equations divide into two types. The system of equations defined by $1 \leq n^{2}<P_{0}$ has a hyperbolic equilibrium at the origin whereas, for the system of equations satisfying $n^{2} \geq P_{0}$, this equilibrium is a center.

For simplicity let us assume $1<P_{0}<4$. Then only the equation with $n=1$ is hyperbolic while the system of remaining equations has a center. To emphasize this let us define $p=u_{1}$ and $q_{n}=u_{n+1}, n=1,2, \ldots$. The preceding equations now take the form 


$$
\begin{gathered}
\ddot{p}=a^{2} p-\frac{\pi}{2}\left[p^{2}+\sum_{k=1}^{\infty}(k+1)^{2} q_{k}^{2}\right] p-2 \mu_{2} \dot{p}+\frac{4}{\pi} \mu_{1} \cos \omega_{0} t, \\
\ddot{q_{n}}=-\omega_{n}^{2} q_{n}-\frac{\pi}{2}(n+1)^{2}\left[p^{2}+\sum_{k=1}^{\infty}(k+1)^{2} q_{k}^{2}\right] q_{n} \\
-2 \mu_{2} \dot{q}_{n}+2 \mu_{1}\left[\frac{1-(-1)^{n+1}}{\pi(n+1)}\right] \cos \omega_{0} t, \\
n=1,2, \ldots
\end{gathered}
$$

where we have defined $a^{2}=P_{0}-1$ and $\omega_{n}^{2}=(n+1)^{2}\left[(n+1)^{2}-P_{0}\right]$.

In (1.2) we project onto the hyperbolic subspace by setting $q=0$ in (1.2a) to obtain what we shall call the reduced equation. In our example this is

$$
\ddot{p}=a^{2} p-\frac{\pi}{2} p^{3}-2 \mu_{2} \dot{p}+\frac{4}{\pi} \mu_{1} \cos \omega_{0} t .
$$

We see that this is the forced, damped Duffing equation with negative stiffness for which standard theory yields chaotic dynamics. The purpose of the present work is to show that the chaotic dynamics of (1.3) are, in some sense, shadowed in the dynamics of the full equation (1.2).

To put our example in first order form we define $x=(p, \dot{p})$ and

$$
y=\left(q_{1}, \dot{q}_{1} / \omega_{1}, q_{2}, \dot{q}_{2} / \omega_{2}, \ldots\right) .
$$

The equations (1.2) now become

$$
\begin{aligned}
\dot{x}_{1}= & x_{2}, \\
\dot{x}_{2}= & a^{2} x_{1}-\frac{\pi}{2}\left[x_{1}^{2}+\sum_{k=1}^{\infty}(k+1)^{2} y_{2 k-1}^{2}\right] x_{1} \\
\dot{y}_{2 n-1}= & \omega_{n} y_{2 n}, \quad-2 \mu_{2} x_{2}+\frac{4}{\pi} \mu_{1} \cos \omega_{0} t \\
\dot{y}_{2 n}= & -\omega_{n} y_{2 n-1}-\frac{\pi}{2} \frac{(n+1)^{2}}{\omega_{n}}\left[x_{1}^{2}+\sum_{k=1}^{\infty}(k+1)^{2} y_{2 k-1}^{2}\right] y_{2 n-1} \\
& -2 \mu_{2} y_{2 n}+2 \mu_{1}\left[\frac{1-(-1)^{n+1}}{\pi(n+1) \omega_{n}}\right] \cos \omega_{0} t .
\end{aligned}
$$

For these equations we define the Hilbert space

$$
\mathbb{Y}=\left\{y=\left\{y_{n}\right\}_{n=1}^{\infty} \mid y_{n} \in \mathbb{R}, \quad \sum_{n=1}^{\infty} \omega_{n}^{2}\left(y_{2 n-1}^{2}+y_{2 n}^{2}\right)<\infty\right\}
$$

with inner product $\langle u, v\rangle=\sum_{n=1}^{\infty} \omega_{n}^{2}\left(u_{2 n-1} v_{2 n-1}+u_{2 n} v_{2 n}\right)$. By a weak solution to (1.4) we mean a pair of functions $x_{0}: \mathbb{R} \rightarrow \mathbb{R}^{2}, y_{0}: \mathbb{R} \rightarrow \mathbb{Y}$ such that $x_{0}$ is differentiable and $y_{0}$ has a derivative $\dot{y}_{0} \rightarrow \ell^{2}$ and which satisfy (1.4a), (1.4b) 
pointwise in $\mathbb{R}^{2} ;(1.4 \mathrm{c}),(1.4 \mathrm{~d})$ pointwise in $\ell^{2}$. Note that in this case we have

$$
\begin{aligned}
& \left(u_{1}, u_{2}, \ldots\right)=\left(x, p_{1}, p_{2}, \ldots\right), \quad x^{2}+\sum_{n=1}^{\infty} \omega_{n}^{2} p_{n}^{2}<\infty, \\
& \left(\dot{u}_{1}, \dot{u}_{2}, \ldots\right)=\left(\dot{x}, \dot{p}_{1}, \dot{p}_{2} \ldots\right) \in \ell^{2}
\end{aligned}
$$

so that for the original differential equation (1.1), $u \in \mathbb{H}^{2}(0, \pi) \cap \mathbb{H}_{0}^{1}(0, \pi)$ and $\dot{u} \in \mathbb{L}^{2}(0, \pi)$. This is discussed in $[\mathbf{1 0}]$.

In the next section we will formulate an abstract problem for which the hypotheses will consist of the essential features of (1.4). We have already mentioned one of these: when $y$ is set equal to zero in (1.4a) the resulting equation is the transverse perturbation of an autonomous equation with a homoclinic solution.

To see another important property we linearize (1.4c), (1.4d) about the origin which yields the system of equations

$$
\left.\begin{array}{rl}
\dot{v}_{2 n-1} & =\omega_{n} v_{2 n} \\
\dot{v}_{2 n} & =-\omega_{n} v_{2 n-1}-2 \mu_{2} v_{2 n}
\end{array}\right\} n=1,2, \ldots .
$$

Note that for each $n$ we get a pair of equations uncoupled from the others and for $\left|\mu_{2}\right|<\omega_{n}$ we have a fundamental solution for $\left(v_{2 n-1}, v_{2 n}\right)$ given by

$$
V_{n}(t)=\left[\begin{array}{cc}
\cos \tilde{\omega}_{n} t+\frac{\mu_{2}}{\tilde{\omega}_{n}} \sin \tilde{\omega}_{n} t & \frac{\omega_{n}}{\tilde{\omega}_{n}} \sin \tilde{\omega}_{n} t \\
-\frac{\omega_{n}}{\tilde{\omega}_{n}} \sin \tilde{\omega}_{n} t & \cos \tilde{\omega}_{n} t-\frac{\mu_{2}}{\tilde{\omega}_{n}} \sin \tilde{\omega}_{n} t
\end{array}\right] \mathrm{e}^{-\mu_{2} t}
$$

where $\tilde{\omega}_{n}=\sqrt{\omega_{n}^{2}-\mu_{2}^{2}}$. This solution has the properties $V_{n}(0)=\mathbb{I}$ and

$$
\left|V_{n}(t) V_{n}(s)^{-1}\right|=\left|V_{n}(t) V_{n}(-s)\right|=\left|V_{n}(t-s)\right| \leq K \mathrm{e}^{\mu_{2}(s-t)}
$$

where $K>0$ is independent of $n$.

Using the sequence $\left\{V_{n}\right\}_{n=1}^{\infty}$ we can define a group $\left\{V_{\mu_{2}}(t)\right\}$ of bounded operators from $\mathbb{Y}$ to $\mathbb{Y}$ by

$$
\left[\begin{array}{l}
\left(V_{\mu_{2}}(t) y\right)_{2 n-1} \\
\left(V_{\mu_{2}}(t) y\right)_{2 n}
\end{array}\right]=V_{n}(t)\left[\begin{array}{l}
y_{2 n-1} \\
y_{2 n}
\end{array}\right] .
$$

Then $\left|V_{\mu_{2}}(t) V_{\mu_{2}}(s)^{-1}\right| \leq K \mathrm{e}^{\mu_{2}(s-t)}$. For $y^{0} \in \mathbb{Y}, y(t)=V_{\mu_{2}}(t) y^{0}$ is the weak solution to (1.5) satisfying $y(0)=y^{0}$.

If we retain the forcing term from (1.4d) we obtain the system of nonhomogeneous variational equations

$$
\begin{aligned}
\dot{v}_{2 n-1} & =\omega_{n} v_{2 n}, \\
\dot{v}_{2 n} & =-\omega_{n} v_{2 n-1}-2 \mu_{2} v_{2 n}+\mu_{1} \nu_{n} \cos \omega_{0} t,
\end{aligned}
$$

where $\nu_{n}=\frac{2\left[1-(-1)^{n+1}\right]}{\pi(n+1) \omega_{n}}$.

Here we encounter the question of resonance. In the nonresonant case, i.e. $\omega_{n} \neq \omega_{0}$, the preceding has a particular solution in $\mathbb{Y}$ with components given by

$$
\left[\begin{array}{l}
v_{2 n-1}(t) \\
v_{2 n}(t)
\end{array}\right]=\frac{\mu_{1} \nu_{n}}{\left(\omega_{n}^{2}-\omega_{0}^{2}\right)^{2}+4 \mu_{2}^{2} \omega_{0}^{2}}\left[\begin{array}{c}
\omega_{n}\left(\omega_{n}^{2}-\omega_{0}^{2}\right) \cos \omega_{0} t+2 \mu_{2} \omega_{0} \omega_{n} \sin \omega_{0} t \\
-\omega_{0}\left(\omega_{n}^{2}-\omega_{0}^{2}\right) \sin \omega_{0} t+2 \mu_{2} \omega_{0}^{2} \cos \omega_{0} t
\end{array}\right]
$$

We make the existence of such a solution a separate hypothesis.

Finally, we mention others works on chaos in partial differential equations. For the complex Ginzburg-Landau equation in the near nonlinear Schrödinger regime (i.e. perturbed nonlinear Schrödinger equation), existence of homoclinic orbits is 
proved by Li, McLaughlin, Shatah and Wiggins $[\mathbf{2 1}, \mathbf{2 7}, \mathbf{2 8}$, and existence of chaos is shown by $\mathrm{Li}[\mathbf{2 2}, \mathbf{2 3}]$ under generic conditions. For perturbed sine-Gordon equation, existence of chaos and chaos cascade around a homoclinic tube was proved by $\operatorname{Li}[\mathbf{2 4}, \mathbf{2 5}, \mathbf{2 6}]$. For the reaction-diffusion equation, entropy study on the complexity of attractor is conducted by Zelik $[\mathbf{3 6}, \mathbf{3 7}, \mathbf{3 8}]$. Chaotic oscillations of a linear wave equation with nonlinear boundary conditions are shown by Chen, Hsu and Zhou [6]. The development of chaos and its controlling for PDEs is summarized by Zhao [39]. Chaos for elastic beams is shown by Battelli and Fečkan [1].

\section{The Abstract Problem}

Using the example in the preceding section as a model we now develop an abstract theory. Let $\mathbb{Y}$ and $\mathbb{H}$ be separable real Hilbert spaces with $\mathbb{Y} \subset \mathbb{H}$.

We now consider differential equations of the form

$$
\begin{aligned}
& \dot{x}=f(x, y, \mu, t)=f_{0}(x, y)+\mu_{1} f_{1}(x, y, \mu, t)+\mu_{2} f_{2}(x, y, \mu, t), \\
& \dot{y}=g(x, y, \mu, t)=A y+g_{0}(x, y)+\mu_{1} \nu \cos \omega_{0} t+\mu_{2} g_{2}(x, y, \mu),
\end{aligned}
$$

with $x \in \mathbb{R}^{n}, y \in \mathbb{Y}, \mu=\left(\mu_{1}, \mu_{2}\right) \in \mathbb{R}^{2}, \nu \in \mathbb{Y}$. We make the following assumptions about (2.1):

(H1) $A: \mathbb{Y} \rightarrow \mathbb{H}$ is a continuous and linear transformation.

(H2) The functions $f_{i}$ and $g_{i}$ are in the spaces:

$$
\begin{aligned}
& f_{0} \in \mathcal{C}^{4}\left(\mathbb{R}^{n} \times \mathbb{Y}, \mathbb{R}^{n}\right) ; \quad f_{1}, f_{2} \in \mathcal{C}^{4}\left(\mathbb{R}^{n} \times \mathbb{Y} \times \mathbb{R}^{2} \times \mathbb{R}, \mathbb{R}^{n}\right) ; \\
& g_{0} \in \mathcal{C}^{4}\left(\mathbb{R}^{n} \times \mathbb{Y}, \mathbb{Y}\right) ; \quad g_{2} \in \mathcal{C}^{4}\left(\mathbb{R}^{n} \times \mathbb{Y} \times \mathbb{R}^{2}, \mathbb{Y}\right) .
\end{aligned}
$$

(H3) $f_{1}$ and $f_{2}$ are periodic in $t$ with period $T=2 \pi / \omega_{0}$.

(H4) $f_{0}(0,0)=0$ and $D_{2} f_{0}(x, 0)=0$.

(H5) The eigenvalues of $D_{1} f_{0}(0,0)$ lie off the imaginary axis.

(H6) The equation $\dot{x}=f_{0}(x, 0)$ has a nontrivial solution homoclinic to $x=0$.

(H7) $g_{0}(x, 0)=g_{2}(x, 0, \mu)=0, D_{12} g_{0}(0,0)=0$ and $D_{22} g_{0}(x, 0)=0$.

(H8) There are constants $K>0, \delta>0$ and $b>0$ so that when $0 \leq\left|\mu_{2}\right| \leq \delta$ the variational equation

$$
\dot{v}=\left(A+\mu_{2} D_{2} g_{2}(0,0,0)\right) v
$$

has a group $\left\{V_{\mu_{2}}(t)\right\}$ of bounded evolution operators from $\mathbb{Y}$ to $\mathbb{Y}$ satisfying $\left|V_{\mu_{2}}(t) V_{\mu_{2}}(s)^{-1}\right| \leq K \mathrm{e}^{b \mu_{2}(s-t)}$.

(H9) There is a constant $K>0$ such that the nonhomogeneous variational equation

$$
\dot{v}=\left[A+\mu_{2} D_{2} g_{2}(0,0,0)\right] v+\mu_{1} \nu \cos \omega_{0} t
$$

has a particular solution $\psi: \mathbb{R} \rightarrow \mathbb{Y}$ satisfying $|\psi(t)| \leq K\left|\mu_{1}\right||\nu|$.

By a weak solution to (2.1) we mean a pair of continuous functions $x_{0}: \mathbb{R} \rightarrow \mathbb{R}^{n}$, $y_{0}: \mathbb{R} \rightarrow \mathbb{Y}$ such that $x_{0}$ is differentiable and $y_{0}$ has a derivative $\dot{y}_{0}: \mathbb{R} \rightarrow \mathbb{H}$ and which satisfy (2.1) pointwise in $\mathbb{H}$.

By (H8) we mean that $V_{\mu_{2}}(s)^{-1}=V_{\mu_{2}}(-s), V_{\mu_{2}}(s) \circ V_{\mu_{2}}(t)=V_{\mu_{2}}(s+t)$, $V_{\mu_{2}}(0)=\mathbb{I}$ and that for $y_{0} \in \mathbb{Y}, y(t)=V_{\mu_{2}}(t) y_{0}$ is the weak solution to $\dot{v}=$ $\left[A+\mu_{2} D_{2} g_{2}(0,0,0)\right] v$ satisfying $y(0)=y_{0}$. 


\section{Chaos on the Hyperbolic Subspace}

The reduced system of equations for (2.1) is

$$
\dot{x}=f(x, 0, \mu, t)=f_{0}(x, 0)+\mu_{1} f_{1}(x, 0, \mu, t)+\mu_{2} f_{2}(x, 0, \mu, t)
$$

with $x \in \mathbb{R}^{n}$. In $[\mathbf{3}, \mathbf{1 3}, \mathbf{1 4}, \mathbf{1 5}]$ a general Melnikov theory is developed for first order systems in $\mathbb{R}^{n}$. We summarize those results here as applied to (3.1).

By (H6), (3.1) has a nontrivial homoclinic solution $\gamma$ when $\mu=0$. By the variational equation along $\gamma$ we mean the linear equation

$$
\dot{u}=D_{1} f_{0}(\gamma, 0) u
$$

and by the adjoint the system

$$
\dot{v}=-D_{1} f_{0}(\gamma, 0)^{*} v .
$$

We let $\left\{u_{1}, \ldots, u_{d}\right\}$ denote a basis for the vector space of bounded solutions to (3.2) with $u_{d}=\dot{\gamma}$ and we let $\left\{v_{1}, \ldots, v_{d}\right\}$ denote a basis for the vector space of bounded solutions to (3.3). Now define the functions $a_{i j}: \mathbb{R} \rightarrow \mathbb{R}$, constants $b_{i j k}$ and function

$$
M: \mathbb{R}^{2} \times \mathbb{R} \times \mathbb{R}^{d-1} \rightarrow \mathbb{R}^{d}
$$

by

$$
\begin{aligned}
a_{i j}(\alpha)= & \int_{-\infty}^{\infty}\left\langle v_{i}(t), f_{j}(\gamma(t), 0,0, t+\alpha)\right\rangle d t ; \\
i=1, \ldots, d ; \quad j=1,2 ; & \\
b_{i j k}= & \int_{-\infty}^{\infty}\left\langle v_{i}, D_{11} f_{0}(\gamma, 0) u_{j} u_{k}\right\rangle d t ; \\
i=1, \ldots, d ; \quad j, k=1, \ldots, d-1 ; & \\
M_{i}(\mu, \alpha, \beta)= & \sum_{j=1}^{2} a_{i j}(\alpha) \mu_{j}+\frac{1}{2} \sum_{j, k=1}^{d-1} b_{i j k} \beta_{j} \beta_{k} ; \quad 1 \leq i \leq d .
\end{aligned}
$$

The function $M$ is our bifurcation function and is used in Theorem 1 below.

Suppose that $(3.2)$ has a $(d-1)$-parameter family of homoclinic orbits given by $t \rightarrow \gamma_{\beta}(t)$ with $\beta \in U_{0}$ where $U_{0}$ is an open neighborhood of the origin in $\mathbb{R}^{d-1}$. Then in (3.4) all $b_{i j k}=0$, the hypotheses of Theorem 1 below cannot be satisfied and an alternate bifurcation function is required.

For each fixed $\beta$ we let $\left\{v_{\beta 1}, \ldots, v_{\beta d}\right\}$ denote a basis for the vector space of bounded solutions to the adjoint equation $\dot{v}=-D_{1} f_{0}\left(\gamma_{\beta}, 0\right)^{*} v$. Without loss of generality we can assume that each $v_{\beta i}$ depends differentially on $\beta$. Now define functions $a_{i j}: \mathbb{R} \times U_{0} \rightarrow \mathbb{R}$ and $M: \mathbb{R}^{2} \times \mathbb{R} \times U_{0} \rightarrow \mathbb{R}^{d}$ by

$$
\begin{aligned}
& a_{i j}(\alpha, \beta)= \int_{-\infty}^{\infty}\left\langle v_{\beta i}(t), f_{j}\left(\gamma_{\beta}(t), 0,0, t+\alpha\right)\right\rangle d t ; \\
& i=1, \ldots, d ; \quad j=1,2 ; \\
& M_{i}(\mu, \alpha, \beta)=\sum_{j=1}^{2} a_{i j}(\alpha, \beta) \mu_{j} ; \quad 1 \leq i \leq d .
\end{aligned}
$$

This function, $M$, is the bifurcation function for this situation.

The concept of exponential dichotomy is important in our next consideration so we state the definition for easy reference (cf. [7]). 
DeFinition 1. We say the linear differential equation $\dot{x}=A_{1}(t) x$ has an exponential dichotomy on $\mathbb{R}$ if its fundamental solution $U$ has a projection $P$ along with constants $(\tilde{A}, a)$ such that:

i): $\left|U(t) P U(s)^{-1}\right| \leq \tilde{A} \mathrm{e}^{a(s-t)}$ for $s \leq t$,

ii): $\left|U(t)(\mathbb{I}-P) U(s)^{-1}\right| \leq \tilde{A} \mathrm{e}^{a(t-s)}$ for $t \leq s$.

The following result can be proved as in $[\mathbf{3}, \mathbf{9}, \mathbf{1 5}, \mathbf{2 9}, \mathbf{3 0}]$.

THEOREM 1. Let $M$ be as in (3.4) or (3.5) and suppose $\mu_{0}, \alpha_{0}, \beta_{0}$ are such that $M\left(\mu_{0}, \alpha_{0}, \beta_{0}\right)=0$ and $D_{(\alpha, \beta)} M\left(\mu_{0}, \alpha_{0}, \beta_{0}\right)$ is nonsingular. Then there exists an interval $J=\left(0, \xi_{0}\right]$ such that for each $\xi \in J$ the equation $\dot{x}=f\left(x, 0, \xi \mu_{0}, t\right)$ has a homoclinic solution $\gamma_{\xi}$ to a small hyperbolic periodic solution.

Furthermore, $\gamma_{\xi}$ depends continuously on $\xi$,

$$
\lim _{\xi \rightarrow 0} \gamma_{\xi}(t)=\gamma\left(t-\alpha_{0}\right) \quad\left(\text { or }=\gamma_{\beta_{0}}\left(t-\alpha_{0}\right), \text { respectively }\right)
$$

uniformly in $t$ and the variational equation along $\gamma_{\xi}$ has an exponential dichotomy on $\mathbb{R}$.

We can use the preceding result to obtain chaos for (3.1) as follows: Let $\Sigma$ denote the space of doubly infinite sequences with entries from the set of integers $\{0,1\}$. The space $\Sigma$, endowed with the metric

$$
\left.\left.d\left(\left\{\sigma_{n}\right\}_{n \in \mathbb{Z}}\right\},\left\{\sigma_{n}^{\prime}\right\}_{n \in \mathbb{Z}}\right\}\right):=\sum_{n \in \mathbb{Z}} \frac{\left|\sigma_{n}-\sigma_{n}^{\prime}\right|}{2^{|n|+1}},
$$

is a compact metric space. Let $\varphi: \Sigma \rightarrow \Sigma$ be the Bernoulli shift map defined by

$$
\varphi\left(\left\{\sigma_{j}\right\}_{j \in \mathbb{Z}}\right)=\left\{\tilde{\sigma}_{j}\right\}_{j \in \mathbb{Z}}, \quad \tilde{\sigma}_{j}=\sigma_{j+1} .
$$

The dynamics of $\varphi$ is extremely rich as it is indicated in the next theorem $[\mathbf{8}, \mathbf{1 9}$, 31, 35].

THEOREM 2. $\varphi$ is a homeomorphism having

i) a countable infinity of periodic orbits of all possible periods,

ii) an uncountable infinity of nonperiodic orbits, and

iii) a dense orbit.

Suppose Theorem 1 holds. Then we can show chaos for the differential equation $\dot{x}=f\left(x, 0, \xi \mu_{0}, t\right)$ by establishing a topological conjugacy between $\varphi$ and some multiple of the period map of the flow for the differential equation $[\mathbf{3 4}, \mathbf{3 5}]$. For this, first, for any $m \in \mathbb{N}, \xi \in J$ and $\sigma \in \Sigma$ define the function $\gamma_{\xi, \sigma, m} \in L^{\infty}\left(\mathbb{R}, \mathbb{R}^{n}\right)$ by

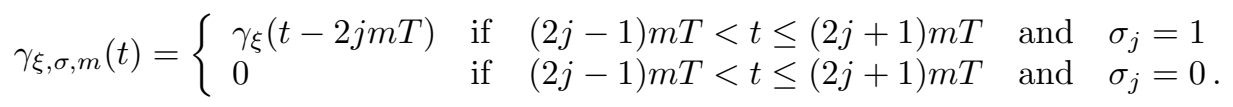

We now use Theorem 1 to show chaos for (3.1) following $[\mathbf{1}, \mathbf{1 1}, \mathbf{2 9}, \mathbf{3 0}]$.

TheOrem 3. a): Let $\mu_{0}, \alpha_{0}, \beta_{0}, \xi_{0}$ be as in Theorem 1. Fix $\xi \in\left(0, \xi_{0}\right]$ and let $\gamma_{\xi}$ be obtained from Theorem 1 . Then there exist an $\varepsilon_{0}>0$ and $a$ function $\varepsilon \rightarrow M(\varepsilon) \in \mathbb{N}$ such that given $\varepsilon$ with $0<\varepsilon \leq \varepsilon_{0}$ and a positive integer $m \geq M(\varepsilon)$ the equation $\dot{x}=f\left(x, 0, \xi \mu_{0}, t\right)$ has for each $\sigma \in \Sigma a$ unique solution $t \rightarrow x_{\sigma}(t)$ satisfying

$$
\left|x_{\sigma}(t)-\gamma_{\xi, \sigma, m}(t)\right| \leq \varepsilon \quad \forall t \in \mathbb{R} .
$$


b): $x_{\sigma}$ depends continuously on $\sigma$ and $x_{\sigma}(t+2 m T)=x_{\varphi(\sigma)}(t)$ where $\varphi$ is the Bernoulli shift on $\Sigma$.

c): The correspondence $\phi(\sigma)=x_{\sigma}(0)$ is a homeomorphism of $\Sigma$ onto the compact subset $\Lambda$ of $\mathbb{R}^{n}$ given by

$$
\Lambda:=\left\{x_{\sigma}(0) \mid \sigma \in \Sigma\right\}
$$

on which the $2 m$ th iterate $F^{2 m}$ of the period map $F$ of (3.1) is invariant and satisfies $F^{2 m} \circ \phi=\phi \circ \varphi$.

Theorem 3 asserts that the following diagram is commutative

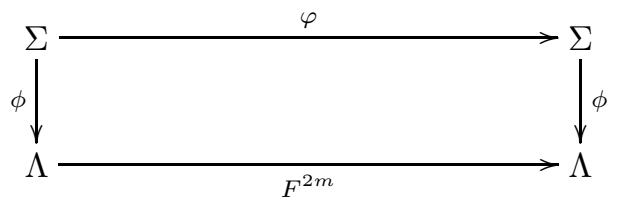

This means that $F^{2 m}: \Lambda \mapsto \Lambda$ has the same dynamics on $\Lambda$ as the Bernoulli shift $\varphi$ on $\Sigma$. Moreover, it is possible to show a sensitive dependence on initial conditions of $F^{2 m}$ on $\Lambda$ in the sense $[\mathbf{8}, \mathbf{3 1}, \mathbf{3 5}]$ that there is an $c_{0}>0$ such that for any $x \in \Lambda$ and any neighborhood $U$ of $x$, there exists $u \in U \cap \Lambda$ and an integer $q \geq 1$ such that

$$
\left|F^{2 m q}(x)-F^{2 m q}(u)\right|>c_{0} .
$$

Consequently, $F^{2 m}$ is chaotic on $\Lambda$, so (3.1) is also chaotic.

This construction is sometimes referred to as embedding a Smale horseshoe in the flow of the differential equation $[\mathbf{8}, \mathbf{3 1}, \mathbf{3 4}]$.

\section{Chaos in the Full Equation}

Since the homoclinic orbit $\gamma_{\xi}$ obtained in Section 3 is hyperbolic the variational equation $\dot{u}=D_{1} f\left(\gamma_{\xi}, 0, \xi \mu_{0}, t\right) u$ has an exponential dichotomy on $\mathbb{R}$ with constant $K_{\xi}$. Now, we show in $[\mathbf{1 2}]$ that the $K_{\xi}$ tends to infinity as $\xi \rightarrow 0$. For this reason we consider the following modification of (2.1)

$$
\begin{aligned}
& \dot{x}=f(x, y, \mu, \lambda, t):=f(x, \lambda y, \mu, t) \\
& \dot{y}=g(x, y, \mu, \lambda, t) \quad:=A y+g_{0}(x, y)+\lambda \mu_{1} \nu \cos \omega_{0} t+\mu_{2} g_{2}(x, y, \mu)
\end{aligned}
$$

for a parameter $\lambda \in[0,1]$.

Now let $\left(\mu_{0}, \alpha_{0}, \beta_{0}\right)$ with $\mu_{0,2} \neq 0$ and $\gamma_{\xi}$ be as in Theorem 1 . Following the arguments of $[\mathbf{1 2}$, pp. $82-85]$, we obtain a constant $\bar{\xi}_{0}$ and for each $\xi \in\left(0, \bar{\xi}_{0}\right]$ a homoclinic orbit

$$
\Gamma(\lambda, \xi)(t)=\left(\Gamma_{1}(\lambda, \xi)(t), \Gamma_{2}(\lambda, \xi)(t)\right)
$$

for (4.1) with $\mu=\xi \mu_{0}$ such that

$$
\begin{array}{ll} 
& \Gamma_{1}(\lambda, \xi)(t) \rightarrow \gamma\left(t-\alpha_{0}\right) \quad\left(\text { or } \rightarrow \gamma_{\beta_{0}}\left(t-\alpha_{0}\right) \text {, respectively }\right), \\
\text { and } \quad & \Gamma_{2}(\lambda, \xi)(t) \rightarrow 0
\end{array}
$$

as $\xi \rightarrow 0$ uniformly for $\lambda \in[0,1]$. Moreover, we have $\Gamma(0, \xi)=\left(\gamma_{\xi}, 0\right)$ and $\Gamma(1, \xi)$ is a homoclinic solution for (2.1). The linearization of (4.1) with $\mu=\xi \mu_{0}$ along $\Gamma(\lambda, \xi)(t)$ has an exponential dichotomy on $\mathbb{R}$ with dichotomy constants uniform with respect to $0 \leq \lambda \leq 1$ and fixed $\xi$. 
Analogous to the construction in Section 3, for each $\sigma \in \Sigma, \xi \in\left(0, \bar{\xi}_{0}\right]$ and $m \in \mathbb{N}$ we construct from $\Gamma(\lambda, \xi)$ a corresponding

$$
\Gamma_{\sigma}(\lambda, \xi, m)=\left(\Gamma_{1, \sigma}(\lambda, \xi, m), \Gamma_{2, \sigma}(\lambda, \xi, m)\right) .
$$

Similarly, from $\gamma_{\xi}$ we obtain $\gamma_{\xi, \sigma, m}$. Then we have $\Gamma_{1, \sigma}(0, \xi, m)=\gamma_{\xi, \sigma, m}$ and also $\Gamma_{2, \sigma}(0, \xi, m)=0$. Using the uniform exponential dichotomy, following $[\mathbf{1}, \mathbf{1 1}]$, we now obtain the following extension of Theorem 3 .

TheOrem 4. a): Let $\mu_{0}, \alpha_{0}, \beta_{0}$ be as in Theorem 1 with $\mu_{0,2} \neq 0$. Fix $\xi \in\left(0, \bar{\xi}_{0}\right]$ and let $\Gamma(\lambda, \xi, m)(t)$ be obtained above. Then there exist an $\bar{\varepsilon}_{0}>0$ and a function $\varepsilon \rightarrow \bar{M}(\varepsilon) \in \mathbb{N}$ such that given $\varepsilon$ with $0<\varepsilon \leq \bar{\varepsilon}_{0}$ and a positive integer $m \geq \bar{M}(\varepsilon)$ the equation (4.1) with $\mu=\xi \mu_{0}$ has for each $\sigma \in \Sigma$ a unique weak solution $t \rightarrow\left(x_{\sigma, \lambda}(t), y_{\sigma, \lambda}(t)\right)$ satisfying

$\left|x_{\sigma, \lambda}(t)-\Gamma_{1, \sigma}(\lambda, \xi, m)(t)\right|+\left|y_{\sigma, \lambda}(t)-\Gamma_{2, \sigma}(\lambda, \xi, m)(t)\right| \leq \varepsilon \quad \forall t \in \mathbb{R}$.

b): The functions $\left(x_{\sigma, \lambda}(t), y_{\sigma, \lambda}(t)\right)$ depend continuously on $\sigma, \lambda$ and we also have $x_{\sigma, \lambda}(t+2 m T)=x_{\varphi(\sigma), \lambda}(t), y_{\sigma, \lambda}(t+2 m T)=y_{\varphi(\sigma), \lambda}(t)$ where $\varphi$ is the Bernoulli shift on $\Sigma$.

c): The correspondence $\phi_{\lambda}(\sigma)=\left(x_{\sigma, \lambda}(0), y_{\sigma, \lambda}(0)\right)$ is a homeomorphism of $\Sigma$ onto the compact subset $\Lambda_{\lambda}$ of $\mathbb{R}^{n} \times \mathbb{Y}$ given by

$$
\Lambda_{\lambda}:=\left\{\left(x_{\sigma, \lambda}(0), y_{\sigma, \lambda}(0)\right) \mid \sigma \in \Sigma\right\}
$$

on which the $2 m$ th iterate $F_{\lambda}^{2 m}$ of the period map $F_{\lambda}$ of (4.1) is invariant and satisfies $F_{\lambda}^{2 m} \circ \phi_{\lambda}=\phi_{\lambda} \circ \varphi$.

d): $\left(x_{\sigma, 0}(t), y_{\sigma, 0}(t)\right)=\left(x_{\sigma}(t), 0\right)$ and $\phi_{0}=\phi$ where $\phi$ is as in Theorem 3 .

Summarizing, we obtain the following main result.

Theorem 5. Suppose (H1)-(H10) hold. Let $M$ be as in (3.4) or (3.5) and suppose $\left(\mu_{0}, \alpha_{0}, \beta_{0}\right)$ are such that $M\left(\mu_{0}, \alpha_{0}, \beta_{0}\right)=0$ and $D_{(\alpha, \beta)} M\left(\mu_{0}, \alpha_{0}, \beta_{0}\right)$ is nonsingular. Then there exists $\bar{\xi}_{0}>0$ such that if $0<\xi \leq \bar{\xi}_{0}$, if the parameters in (2.1) are given by $\mu=\xi \mu_{0}$, and $\mu_{0,2} \neq 0$ then there exists a homeomorphism, $\phi_{1}$, of $\Sigma$ onto a compact subset of $\mathbb{R}^{n} \times \mathbb{Y}$ on which the 2 mth iterate, $F_{1}^{2 m}$, of the period map $F_{1}$ of (2.1) is invariant and satisfies $F_{1}^{2 m} \circ \phi_{1}=\phi_{1} \circ \varphi$ where $\varphi$ is the Bernoulli shift on $\Sigma$.

We might paraphrase Theorem 5, loosely, as saying that the Smale horseshoe embedded in the flow of the reduced equation (3.1) is shadowed by a horseshoe in the full equation (2.1).

\section{Applications: Vibrating Elastic Beams}

We now return to the example in Section 1 and apply our theory to the problem of vibrating elastic beams. We shall consider a number of different cases and generalizations. In each case our procedure will be:

i) Use a Galerkin expansion to convert the partial differential equation to an infinite set of ordinary differential equations as (2.1).

ii) Truncate the equation to get the finite problem (3.1).

iii) Apply Theorem 3 to get a Smale horseshoe for the finite problem. For this we must verify (H1) through (H6).

iv) Use Theorem 5 to lift the horseshoe to the flow of the original partial differential differential equation. This requires (H7)-(H9). 
5.1. Planer Motion with One Buckled Mode. The boundary value problem for planer deflections of an elastic beam with a compressive axial load $P_{0}$ and pinned ends is

$$
\begin{aligned}
& \ddot{u}=-u^{\prime \prime \prime \prime}-P_{0} u^{\prime \prime}+\left[\int_{0}^{\pi} u^{\prime}(s)^{2} d s\right] u^{\prime \prime}-2 \mu_{2} \dot{u}+\mu_{1} \cos \omega_{0} t, \\
& u(0, t)=u(\pi, t)=u^{\prime \prime}(0, t)=u^{\prime \prime}(\pi, t)=0
\end{aligned}
$$

where $u(x, t)$ is the transverse deflection at a distance $x$ from one end at time $t$. We consider the $\mu_{i}$ terms as perturbations.

Our first step is to consider the linearized, unperturbed problem. We compute the eigenvalues at the origin to be $\lambda_{n}=n^{2}\left(n^{2}-P_{0}\right)$ with corresponding eigenfunctions $\varphi_{n}(x)=\sin n x$ for $n=1,2, \ldots$. For small $P_{0}$ the origin is a center. As $P_{0}$ is increased the first bifurcation occurs at $P_{0}=1$, the first Euler buckling load. The corresponding eigenfunction, $\varphi_{1}(x)=\sin x$, is referred to as the first buckled mode. The second bifurcation occurs at $P_{0}=4$. Thus, the simplest case, which we now consider, consists of $1<P_{0}<4$.

In the first equation we define $a^{2}=\lambda_{1}=P_{0}-1$. The eigenvalues for the center modes, or unbuckled modes, provide the frequencies used in (2.1) as we define $\omega_{n-1}^{2}=\lambda_{n}=n^{2}\left[n^{2}-P_{0}\right], \quad n=2,3, \ldots$. We now use the eigenfunctions for the Galerkin expansion $u(x, t)=\sum_{k=1}^{\infty} u_{k}(t) \sin k x$ and obtain the system of equations

$$
\begin{aligned}
\ddot{u}_{n}=n^{2}\left(P_{0}\right. & \left.-n^{2}\right) u_{n}-\frac{\pi}{2} n^{2}\left[\sum_{k=1}^{\infty} k^{2} u_{k}^{2}\right] u_{n} \\
& -2 \mu_{2} \dot{u}_{n}+2 \mu_{1}\left[\frac{1-(-1)^{n}}{\pi n}\right] \cos \omega_{0} t, \quad n=1,2, \ldots .
\end{aligned}
$$

To obtain a first order system as in (2.1) we define

$$
x=\left(u_{1}, \dot{u}_{1}\right), \quad y=\left(u_{2}, \dot{u}_{2} / \omega_{1}, u_{3}, \dot{u}_{3} / \omega_{2}, \ldots\right) .
$$

The reduced equations are

$$
\begin{aligned}
& \dot{x}_{1}=x_{2}, \\
& \dot{x}_{2}=a^{2} x_{1}-\frac{\pi}{2} x_{1}^{3}-2 \mu_{2} x_{2}+\frac{4}{\pi} \mu_{1} \cos \omega_{0} t
\end{aligned}
$$

obtained by setting $y=0$ in the hyperbolic part. When $\mu=0,(5.2)$ has a homoclinic solution given by $\gamma=(r, \dot{r})$ where $r(t)=(2 a / \sqrt{\pi})$ sech at. Equation (3.3) becomes

with solution $\left(v_{1}, v_{2}\right)=(-\ddot{r}, \dot{r})$.

$$
\dot{v}_{1}=-\left(a^{2}-\frac{3 \pi}{2} r^{2}\right) v_{2}, \quad \dot{v}_{2}=-v_{1}
$$

In (3.4) we have $d=1$ so the variable $\beta$ does not appear, $M$ is a scalar function, and the function $M=M_{1}$ becomes

$$
M(\alpha)=\left[\frac{8 \omega_{0}}{\sqrt{\pi}} \sin \omega_{0} \alpha \operatorname{sech} \frac{\pi \omega_{0}}{2 a}\right] \mu_{1}-\left(\frac{16 a^{3}}{3 \pi}\right) \mu_{2} .
$$

Thus, the conditions $M\left(\mu_{0}, \alpha_{0}\right)=0,(\partial M / \partial \alpha)\left(\mu_{0}, \alpha_{0}\right) \neq 0$ are satisfied for all $\mu_{0}$ such that

$$
\left|\frac{\mu_{0,2}}{\mu_{0,1}}\right|<\frac{3 \sqrt{\pi} \omega_{0}}{2 a^{3}} \operatorname{sech} \frac{\pi \omega_{0}}{2 a} .
$$




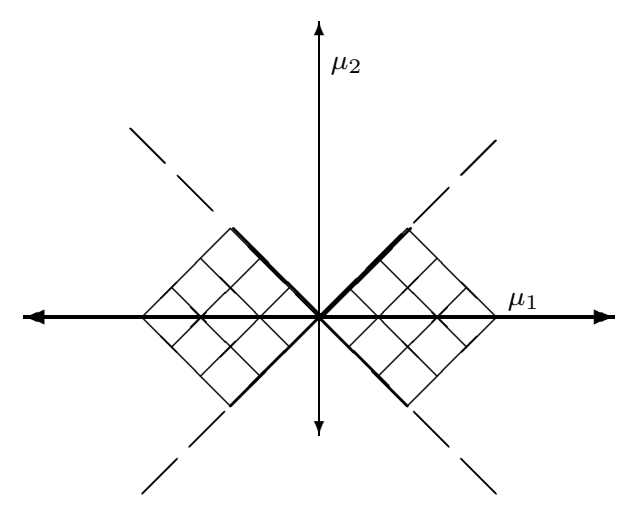

Figure 2. The chaotic open wedge-shaped region of (5.1) in $\mathbb{R}^{2}$.

Now we check condition (H9) which, for the present problem, requires us to consider the equation

$$
\begin{aligned}
\dot{v}_{2 n-1} & =\omega_{n} v_{2 n}, \\
\dot{v}_{2 n} & =-\omega_{n} v_{2 n-1}-2 \mu_{2} v_{2 n}+\mu_{1} \nu_{n} \cos \omega_{0} t
\end{aligned}
$$

where $\nu_{n}=\frac{2\left[1-(-1)^{n-1}\right]}{\pi(n+1) \omega_{n}}$.

This system has a particular solution in $\mathbb{Y}$ with components given by

$$
\left[\begin{array}{l}
v_{2 n-1}(t) \\
v_{2 n}(t)
\end{array}\right]=\frac{\mu_{1} \nu_{n}}{\left(\omega_{n}^{2}-\omega_{0}^{2}\right)^{2}+4 \mu_{2}^{2} \omega_{0}^{2}}\left[\begin{array}{c}
\omega_{n}\left(\omega_{n}^{2}-\omega_{0}^{2}\right) \cos \omega_{0} t+2 \mu_{2} \omega_{0} \omega_{n} \sin \omega_{0} t \\
-\omega_{0}\left(\omega_{n}^{2}-\omega_{0}^{2}\right) \sin \omega_{0} t+2 \mu_{2} \omega_{0}^{2} \cos \omega_{0} t
\end{array}\right] .
$$

From this we see that (H9) is satisfied whenever $\omega_{0} \neq \omega_{n}$ for all $n$.

We note that while the conditions $M(\alpha)=0, M^{\prime}(\alpha) \neq 0$ can be satisfied with $\mu_{2}=0, \alpha=0$ we require $\mu_{2} \neq 0$ in Section 4 where we use a weak exponential dichotomy to lift to the full equation. Thus, we obtain the following result using Theorem 5 .

THEOREM 6. If $\omega_{0} \neq \omega_{n}$ for all $n$ then whenever $\mu_{0}$ satisfies $\mu_{0,1} \neq 0$ and

$$
0<\left|\frac{\mu_{0,2}}{\mu_{0,1}}\right|<\frac{3 \sqrt{\pi} \omega_{0}}{2 a^{3}} \operatorname{sech} \frac{\pi \omega_{0}}{2 a},
$$

there exists a corresponding $\bar{\xi}_{0}>0$ such that if $0<\xi \leq \bar{\xi}_{0}$, if the parameters in (5.1) are given by $\mu=\xi \mu_{0}$ then there exists a compact subset of $\mathbb{R}^{2} \times \mathbb{Y}$ on which the $2 m$ th iterate, $F^{2 m}$, of the period map $F$ of (5.1) is invariant and conjugate to the Bernoulli shift on $\Sigma$.

These results are stated in terms of the Galerkin equations (5.1) but they can be transferred back to the original partial differential equation. In this case we get a Bernoulli shift embedded in $\left[\mathbb{H}_{0}^{1}(0, \pi) \cap \mathbb{H}^{2}(0, \pi)\right] \times \mathbb{L}^{2}(0, \pi)$. This is discussed in [10]. In the $\mu_{1}-\mu_{2}$ plane we get from the condition (5.3) four small open wedge-shaped regions of parameter values for which the partial differential equation exhibits chaos (see Figure 2). These regions are bounded by the lines $\mu_{1} / \mu_{2}= \pm \frac{3 \sqrt{\pi} \omega_{0}}{2 a^{3}} \operatorname{sech} \frac{\pi \omega_{0}}{2 a}$ and $\mu_{2}=0$ 
It is interesting to look at some history of this problem. The first work was by Holmes [17] in which he started with the partial differential equation and carried out the Galerkin expansion but restricted his analysis to the reduced equation (5.2). The significance of that work is that it introduced the idea of Melnikov analysis. In subsequent work [18] Holmes and Marsden extended the results to infinite dimension but abandoned the Galerkin approach in favor of nonlinear semigroup techniques directly in infinite dimensions. In our work we go back to the original, simpler analysis of the reduced equation and then show that the results apply to the original partial differential equation. Some advantages to this are that the Galerkin projection is a technique familiar to many engineers and physicists and, also, we are able to utilize our general Melnikov results in Section 3. This is illustrated further in the generalizations which follow. We note that equation (5.1) was treated also in $[4]$.

5.2. Nonplaner Motion of a Symmetric Beam with One Buckled Mode. Let us consider a beam with symmetric cross section, pinned ends and compressive axial load $P_{0}$ and assume now that the beam is not constrained to defect in a plane. If $u(x, t)$ and $w(x, t)$ denote the transverse defections at position $x$ and time $t$ we obtain the following boundary value problem.

$$
\begin{aligned}
\ddot{u} & =-u^{\prime \prime \prime \prime}-P_{0} u^{\prime \prime}+\left[\int_{0}^{\pi}\left(u^{\prime}(s)^{2}+w^{\prime}(s)^{2}\right) d s\right] u^{\prime \prime} \\
& -2 \mu_{2} \dot{u} \cos \eta+\mu_{1} \cos \zeta \cos \omega_{0} t \\
\ddot{w} & =-w^{\prime \prime \prime \prime}-P_{0} w^{\prime \prime}+\left[\int_{0}^{\pi}\left(u^{\prime}(s)^{2}+w^{\prime}(s)^{2}\right) d s\right] w^{\prime \prime} \\
- & 2 \mu_{2} \dot{w} \sin \eta+\mu_{1} \sin \zeta \cos \omega_{0} t \\
u(0, t) & =u(\pi, t)=u^{\prime \prime}(0, t)=u^{\prime \prime}(\pi, t)=w(0, t) \\
& =w(\pi, t)=w^{\prime \prime}(0, t)=w^{\prime \prime}(\pi, t)=0
\end{aligned}
$$

where $\eta, \zeta$ are constants.

The parameters $\mu_{1}, \mu_{2}$ represent the coefficients of, respectively, total transverse forcing and total viscous damping. These effects are distributed between the two directions of motion. The quantity $\tan \zeta$ represents the ratio of forcing in the $u$-direction to forcing in the $w$-direction while tan $\eta$ plays the same role for the damping. We suppose $\eta, \zeta \in(0, \pi / 2)$ in order to avoid certain degeneracies.

In these equations we use the Galerkin expansions

$$
u(x, t)=\sum_{k=1}^{\infty} u_{k}(t) \sin k x, \quad w(x, t)=\sum_{k=1}^{\infty} w_{k}(t) \sin k x
$$

and proceed as before. This yields the system of equations

$$
\begin{aligned}
\ddot{u}_{n}=n^{2}\left(P_{0}-n^{2}\right) u_{n}-\frac{\pi}{2} n^{2}\left[\sum_{k=1}^{\infty} k^{2}\left(u_{k}^{2}+w_{k}^{2}\right)\right] u_{n} \\
-2 \mu_{2} \dot{u}_{n} \cos \eta+2 \mu_{1} \cos \zeta\left[\frac{1-(-1)^{n}}{\pi n}\right] \cos \omega_{0} t \\
\ddot{w}_{n}=n^{2}\left(P_{0}-n^{2}\right) w_{n}-\frac{\pi}{2} n^{2}\left[\sum_{k=1}^{\infty} k^{2}\left(u_{k}^{2}+w_{k}^{2}\right)\right] w_{n} \\
-2 \mu_{2} \dot{w}_{n} \sin \eta+2 \mu_{1} \sin \zeta\left[\frac{1-(-1)^{n}}{\pi n}\right] \cos \omega_{0} t
\end{aligned}
$$


As before, we assume $1<P_{0}<4$ and define $a^{2}=P_{0}-1$ and

$$
\omega_{n-1}^{2}=n\left(n^{2}-P_{0}\right), n=2,3, \ldots \text {. }
$$

The equations (5.4) take the form of (2.1) when we define $x=\left(u_{1}, \dot{u}_{1}, w_{1}, \dot{w}_{1}\right)$ and $y=\left(u_{2}, \dot{u}_{2} / \omega_{1}, w_{2}, \dot{w}_{2} / \omega_{1}, u_{3}, \dot{u}_{3} / \omega_{2}, w_{3}, \dot{w}_{3} / \omega_{2}, \ldots\right)$.

The reduced equations are

$$
\begin{aligned}
& \dot{x}_{1}=x_{2}, \\
& \dot{x}_{2}=a^{2} x_{1}-\frac{\pi}{2}\left(x_{1}^{2}+x_{3}^{2}\right) x_{1}-2 \mu_{2} x_{2} \cos \eta+\frac{4}{\pi} \mu_{1} \cos \zeta \cos \omega_{0} t, \\
& \dot{x}_{3}=x_{4} \\
& \dot{x}_{4}=a^{2} x_{3}-\frac{\pi}{2}\left(x_{1}^{2}+x_{3}^{2}\right) x_{3}-2 \mu_{2} x_{4} \sin \eta+\frac{4}{\pi} \mu_{1} \sin \zeta \cos \omega_{0} t .
\end{aligned}
$$

When $\mu=0$ we have a two-dimensional homoclinic manifold given by $\gamma_{\beta}=$ $(r \cos \beta, \dot{r} \cos \beta, r \sin \beta, \dot{r} \sin \beta)$ where, as before, $r(t)=(2 a / \sqrt{\pi}) \operatorname{sech} a t$ and $\beta$ is a parameter. The adjoint equations (3.3) take the form

$$
\begin{aligned}
& \dot{v}_{1}=\left[-a^{2}+\frac{\pi}{2}\left(3 r^{2} \cos ^{2} \beta+r^{2} \sin ^{2} \beta\right)\right] v_{2}+\left(\pi r^{2} \sin \beta \cos \beta\right) v_{4}, \\
& \dot{v}_{2}=-v_{1}, \\
& \dot{v}_{3}=\left(\pi r^{2} \sin \beta \cos \beta\right) v_{2}+\left[-a^{2}+\frac{\pi}{2}\left(r^{2} \cos ^{2} \beta+3 r^{2} \sin ^{2} \beta\right)\right] v_{4}, \\
& \dot{v}_{4}=-v_{3} .
\end{aligned}
$$

A one-parameter family of bounded solutions to these equations is given by

$$
\begin{aligned}
& v_{\beta 1}=(-\dot{r} \sin \beta, r \sin \beta, \dot{r} \cos \beta,-r \cos \beta), \\
& v_{\beta 2}=(-\ddot{r} \cos \beta, \dot{r} \cos \beta,-\ddot{r} \sin \beta, \dot{r} \sin \beta)
\end{aligned}
$$

and the function, $M$, as in (3.5) becomes

$$
\begin{aligned}
& M_{1}(\mu, \alpha, \beta)= {\left[\frac{8}{\sqrt{\pi}} \sin (\beta-\zeta) \cos \omega_{0} \alpha \operatorname{sech} \frac{\pi \omega_{0}}{2 a}\right] \mu_{1}, } \\
& M_{2}(\mu, \alpha, \beta)=\left[\frac{8 \omega_{0}}{\sqrt{\pi}} \cos (\beta-\zeta) \sin \omega_{0} \alpha \operatorname{sech} \frac{\pi \omega_{0}}{2 a}\right] \mu_{1} \\
&-\left[\frac{16 a^{3}\left(\cos \eta \cos ^{2} \beta+\sin \eta \sin ^{2} \beta\right)}{3 \pi}\right] \mu_{2} .
\end{aligned}
$$

Next, the conditions $M\left(\mu_{0}, \alpha_{0}, \beta_{0}\right)=0, D_{(\alpha, \beta)} M\left(\mu_{0}, \alpha_{0}, \beta_{0}\right)$ nonsingular are satisfied in two different cases. Of course, we suppose $\mu_{0,1} \neq 0, \mu_{0,2} \neq 0$ and then put $\lambda_{0}=\frac{\mu_{0,2}}{\mu_{0,1}}$. We have the following two cases:

Case 1. We can choose either $\beta_{0}=\zeta$ and then look for a simple root of the equation

$$
\lambda_{0}=m_{1} \sin \omega_{0} \alpha,
$$

or choose $\beta_{0}=\zeta+\pi$ and look for a simple root of the equation

$$
\lambda_{0}=-m_{1} \sin \omega_{0} \alpha
$$

for

$$
m_{1}=\frac{3 \sqrt{\pi} \omega_{0}}{2 a^{2}\left(\cos \eta \cos ^{2} \zeta+\sin \eta \sin ^{2} \zeta\right)} \operatorname{sech} \frac{\pi \omega_{0}}{2 a} .
$$


Supposing the condition

$$
0<\left|\lambda_{0}\right|<m_{1}
$$

there is a simple root $\alpha_{0}$ of (5.6). Similarly, (5.7) has also a simple root $-\alpha_{0}$. According to the formulas (5.5) for $v_{\beta_{1}}$ and $v_{\beta_{2}}$, these simple roots $\left(\zeta, \alpha_{0}\right)$ and $\left(\zeta+\pi,-\alpha_{0}\right)$ give two different solutions of (5.4).

Case 2. In this case we begin by choosing $\omega_{0} \alpha_{0}=\left(2 k_{0}+1\right) \frac{\pi}{2}$ for $k_{0} \in\{0,1\}$ and then we look for a simple root $\beta_{0} \neq \zeta+k \pi, \forall k \in \mathbb{Z}$ of

$$
\lambda_{0}=(-1)^{k_{0}} \Phi(\beta)
$$

where

$$
\Phi(\beta)=\frac{3 \omega_{0} \sqrt{\pi}}{2 a^{3}} \frac{\cos (\beta-\zeta)}{\cos \eta \cos ^{2} \beta+\sin \eta \sin ^{2} \beta} \operatorname{sech} \frac{\pi \omega_{0}}{2 a} .
$$

Let $m_{2}=\max _{\beta \in \mathbb{R}} \Phi(\beta)$. We discuss the computation of the constant $m_{2}$ in Appendix A. Since $\Phi(\beta+\pi)=-\Phi(\beta)$, the range of $\Phi$ is the closed interval $\left[-m_{2}, m_{2}\right]$. We now split this case into two parts:

Part 2A). For $\eta=\pi / 4$ we get $\Phi(\beta)=m_{1} \cos (\beta-\zeta)$, so $m_{2}=m_{1}$. Equation (5.9) has now the form

$$
(-1)^{k_{0}} m_{1} \cos (\beta-\zeta)=\lambda_{0},
$$

so under condition (5.8), there is a simple root $\beta_{0}$ different from $\zeta+k \pi, \forall k \in \mathbb{Z}$. This holds for both cases $k_{0} \in\{0,1\}$ so we have two different solutions of (5.4). In addition, the results of Case 1 still apply here.

Thus, in this situation, we have in the $\mu_{1}-\mu_{2}$ plane four wedged-shaped regions of parameter values bounded by $\mu_{2} / \mu_{1}= \pm m_{1}, \mu_{2}=0$ for which the partial differential equation exhibits chaos. In particular, (5.4) has four distinct homoclinic solutions, two from Case 1, two from Case 2A. These regions are labeled $I I$ in Figure 3. In this case there are no regions labeled $I$.

Part 2B). For $\eta \neq \pi / 4$ we get $\Phi^{\prime}(\zeta) \neq 0$, so $m_{1}<m_{2}$. Certainly for the solvability of (5.9) we need $\left|\lambda_{0}\right| \leq m_{2}$. Now we claim:

LEMma 1 . If

$$
\lambda_{0} \in\left(-m_{2}, m_{2}\right) \backslash\left\{ \pm m_{1}, 0\right\},
$$

then equation (5.9) has a simple root $\beta_{0} \in[0,2 \pi] \backslash\{\zeta, \zeta+\pi\}$.

Proof. Assume to the contrary that (5.9) has no simple roots for a $\lambda_{0} \in$ $\left(-m_{2}, m_{2}\right) \backslash\left\{ \pm m_{1}, 0\right\}$. Then there are $0 \leq \beta_{1}<\beta_{2} \leq 2 \pi$ such that

$$
\Phi\left(\beta_{1,2}\right)=(-1)^{k_{0}} \lambda_{0}, \quad \Phi^{\prime}\left(\beta_{1,2}\right)=0, \quad \Phi^{\prime \prime}\left(\beta_{1,2}\right)=0 .
$$

Note then $\beta_{1,2} \neq \zeta+k \pi$ and $\beta_{1,2} \neq \zeta+\frac{2 k+1}{2} \pi, \forall k \in\{0,1\}$. After some calculation we derive from (5.11) that $\cos 2 \beta_{1,2} \neq 0, \sin 2 \beta_{1,2} \neq 0$ and that (5.11) is equivalent to

$$
\begin{aligned}
\frac{\cos \left(\beta_{1,2}-\zeta\right)}{\cos \eta \cos ^{2} \beta_{1,2}+\sin \eta \sin ^{2} \beta_{1,2}} & =\frac{\sin \left(\beta_{1,2}-\zeta\right)}{(\cos \eta-\sin \eta) \sin 2 \beta_{1,2}} \\
=\frac{\cos \left(\beta_{1,2}-\zeta\right)}{2(\cos \eta-\sin \eta) \cos 2 \beta_{1,2}} & =(-1)^{k_{0}} \frac{2 a^{3}}{3 \omega_{0} \sqrt{\pi}} \cosh \frac{\pi \omega_{0}}{2 a} \lambda_{0} .
\end{aligned}
$$




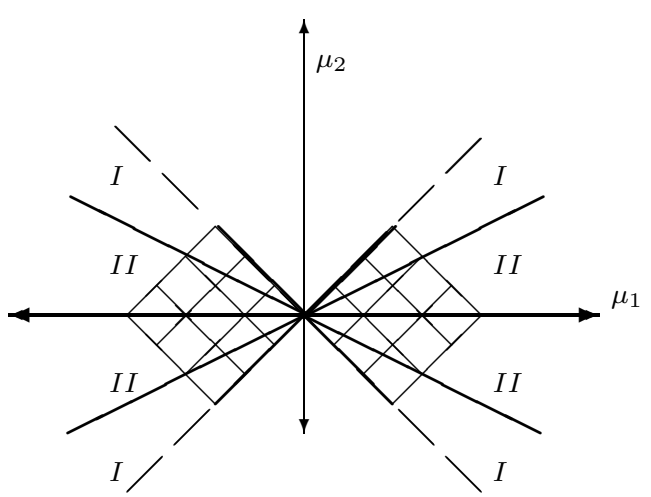

Figure 3. The chaotic wedge-shaped regions of (5.4) in $\mathbb{R}^{2}$.

From (5.12) we derive

$$
\cos 2 \beta_{1,2}=\frac{\cos \eta+\sin \eta}{3(\cos \eta-\sin \eta)}, \quad 2 \tan \left(\beta_{1,2}-\zeta\right)=\tan 2 \beta_{1,2} .
$$

Hence

$$
\beta_{2} \in\left\{\pi-\beta_{1}, \pi+\beta_{1}, 2 \pi-\beta_{1}\right\} .
$$

If $\beta_{2}=\pi-\beta_{1}$ then from $2 \tan \left(\beta_{2}-\zeta\right)=\tan 2 \beta_{2}$ we get $2 \tan \left(\beta_{1}+\zeta\right)=\tan 2 \beta_{1}$, but $2 \tan \left(\beta_{1}-\zeta\right)=\tan 2 \beta_{1}$, so

$$
\tan \left(\beta_{1}+\zeta\right)=\tan \left(\beta_{1}-\zeta\right)
$$

i.e. $\zeta=k \pi / 2, k \in\{0,1\}$. This contradicts $\zeta \in(0, \pi / 2)$.

If $\beta_{2}=\pi+\beta_{1}$ then

$$
(-1)^{k_{0}} \lambda_{0}=\Phi\left(\beta_{2}\right)=\Phi\left(\beta_{1}+\pi\right)=-\Phi\left(\beta_{1}\right)=(-1)^{k_{0}+1} \lambda_{0},
$$

which implies $\lambda_{0}=0$, a contradiction.

If $\beta_{2}=2 \pi-\beta_{1}$ then again we derive $\tan \left(\beta_{1}+\zeta\right)=\tan \left(\beta_{1}-\zeta\right)$, so that $\zeta=k \pi / 2$, $k \in\{0,1\}$, a contradiction to $\zeta \in(0, \pi / 2)$. The proof is finished.

Note $\beta_{0} \in\{\zeta, \zeta+\pi\}$ for the Case 1 , while $\beta_{0} \in[0,2 \pi) \backslash\{\zeta, \zeta+\pi\}$ for the Case 2. Lemma 1 can be applied to both cases $\alpha_{0}=\frac{\pi}{2 \omega_{0}}\left(2 k_{0}+1\right), k_{0} \in\{0,1\}$ so Part 2B yields, in the $\mu_{1}-\mu_{2}$ plane, four wedge-shaped regions of parameter values bounded by $\mu_{2} / \mu_{1}= \pm m_{2}, \mu_{2} / \mu_{1}= \pm m_{1}, \mu_{2}=0$ for which (5.4) has two different homoclinic solutions. These regions are labeled $I$ in Figure 3. Note we have four different solutions of (5.4) on regions labeled $I I$, since there the Case 1 can be also applied (see (5.6) and (5.7)). This completes the analysis of the Melnikov function.

We now check for resonance. Because in the present problem all coupling terms are nonlinear, the linear equation in (H9) consists in two copies of the system of equations in the preceding example. This yields the following result obtained from Theorem 5.

Theorem 7. Suppose $\omega_{0} \neq \omega_{n}$ for all $n$ and let $m_{1}, m_{2}$ be as above. 
i) If $m_{0} \neq 0$ satisfies one but not both of $\left|m_{0}\right|<m_{i}$ then if $\mu_{0,2} / \mu_{0,1}=$ $m_{0}$ there exists a corresponding $\bar{\xi}_{0}>0$ such that if $0<\xi \leq \bar{\xi}_{0}$, if the parameters in (5.4) are given by $\mu=\xi \mu_{0}$ then there exist two homoclinic orbits which can be used to construct a compact subset of $\mathbb{R}^{4} \times \mathbb{Y}$ on which the 2 mth iterate, $F^{2 m}$, of the period map $F$ of (5.4) is invariant and conjugate to the Bernoulli shift on $\Sigma$.

ii) If $m_{0} \neq 0$ satisfies each of $\left|m_{0}\right|<m_{i}$ then there are four homoclinic orbits as in (i).

Summarizing, we obtain eight open small wedge-shaped regions of parameter values in the $\mu_{1}-\mu_{2}$ plane bounded by the lines $\mu_{2} / \mu_{1}= \pm m_{1}, \mu_{2} / \mu_{1}= \pm m_{2}$ and $\mu_{2}=0$ with $m_{1} \leq m_{2}$ for which the partial differential equation exhibits chaos (see Figure 3 ). In the regions labeled $I$ there are two homoclinics while in regions $I I$ there exist four. It is interesting to note that in this case, by adjusting the parameters $\eta$ and $\zeta$, it is possible to make the size of the wedge arbitrarily close to filling the $\mu_{1}-\mu_{2}$ plane.

5.3. Nonplaner, Nonsymmetric Beam with One Buckled Mode in Each Plane. For the case of a nonsymmetric beam with nonplaner motion we have the boundary value problem

$$
\begin{gathered}
\ddot{u}=-u^{\prime \prime \prime \prime}-P_{0} u^{\prime \prime}+\left[\int_{0}^{\pi}\left(u^{\prime}(s)^{2}+w^{\prime}(s)^{2}\right) d s\right] u^{\prime \prime} \\
-2 \mu_{2} \dot{u} \cos \eta+\mu_{1} \cos \zeta \cos \omega_{0} t \\
\ddot{w}=-R^{2} w^{\prime \prime \prime \prime}-P_{0} w^{\prime \prime}+\left[\int_{0}^{\pi}\left(u^{\prime}(s)^{2}+w^{\prime}(s)^{2}\right) d s\right] w^{\prime \prime} \\
-2 \mu_{2} \dot{w} \sin \eta+\mu_{1} \sin \zeta \cos \omega_{0} t \\
u(0, t)=u(\pi, t)=u^{\prime \prime}(0, t)=u^{\prime \prime}(\pi, t) \\
=w(0, t)=w(\pi, t)=w^{\prime \prime}(0, t)=w^{\prime \prime}(\pi, t)=0
\end{gathered}
$$

where $R^{2}$ is constant representing the stiffness ratio for the two directions. We assume $R>1$ which amounts to choosing $w$ as the direction with stiffer crosssection. Note that $R=1$ reduces to Section 5.2. As before we assume $\eta, \zeta \in$ $(0, \pi / 2)$.

The Galerkin expansion becomes

$$
\begin{gathered}
\ddot{u}_{n}=n^{2}\left(P_{0}-n^{2}\right) u_{n}-\frac{\pi}{2} n^{2}\left[\sum_{k=1}^{\infty} k^{2}\left(u_{k}^{2}+w_{k}^{2}\right)\right] u_{n} \\
-2 \mu_{2} \dot{u}_{n} \cos \eta+2 \mu_{1} \cos \zeta\left[\frac{1-(-1)^{n}}{\pi n}\right] \cos \omega_{0} t, \\
\ddot{w}_{n}=n^{2}\left(P_{0}-n^{2} R^{2}\right) w_{n}-\frac{\pi}{2} n^{2}\left[\sum_{k=1}^{\infty} k^{2}\left(u_{k}^{2}+w_{k}^{2}\right)\right] w_{n} \\
-2 \mu_{2} \dot{w}_{n} \sin \eta+2 \mu_{1} \sin \zeta\left[\frac{1-(-1)^{n}}{\pi n}\right] \cos \omega_{0} t .
\end{gathered}
$$

If $P_{0}$ is increased only enough to give one buckled mode, necessarily in the $u$ direction, the problem reduces to Section 5.1. We shall assume here the next simplest case consisting of one buckled mode in each direction which occurs when $1<P_{0}<4$ 
and $R^{2}<P_{0}<4 R^{2}$. Note that this requires $R<2$ and we assume $R^{2}<P_{0}<4$. If the stiffness ratio is too high there will be multiple buckled in the $u$ (soft) direction before occurrence of the first buckled mode in the $w$ (stiff) direction.

We define

$$
\begin{aligned}
& a_{1}^{2}=P_{0}-1, \quad \omega_{n-1,1}^{2}=n^{2}\left[\left(n^{2}-P_{0}\right], \quad n=2,3, \ldots ;\right. \\
& a_{2}^{2}=P_{0}-R^{2}, \quad \omega_{n-1,2}^{2}=n^{2}\left[n^{2} R^{2}-P_{0}\right], \quad n=2,3, \ldots .
\end{aligned}
$$

We put (5.14) in the form of (2.1) by defining

$$
\begin{aligned}
& x=\left(u_{1}, \dot{u}_{1}, w_{1}, \dot{w}_{1}\right), \\
& y=\left(u_{2}, \dot{u}_{2} / \omega_{1,1}, w_{2}, \dot{w}_{2} / \omega_{1,2}, u_{3}, \dot{u}_{3} / \omega_{2,1}, w_{3}, \dot{w}_{3} / \omega_{2,2}, \ldots\right) .
\end{aligned}
$$

The reduced equations are

$$
\begin{aligned}
& \dot{x}_{1}=x_{2}, \\
& \dot{x}_{2}=a_{1}^{2} x_{1}-\frac{\pi}{2}\left(x_{1}^{2}+x_{3}^{2}\right) x_{1}-2 \mu_{2} x_{2} \cos \eta+\frac{4}{\pi} \mu_{1} \cos \zeta \cos \omega_{0} t, \\
& \dot{x}_{3}=x_{4} \\
& \dot{x}_{4}=a_{2}^{2} x_{3}-\frac{\pi}{2}\left(x_{1}^{2}+x_{3}^{2}\right) x_{3}-2 \mu_{2} x_{4} \sin \eta+\frac{4}{\pi} \mu_{1} \sin \zeta \cos \omega_{0} t .
\end{aligned}
$$

For the unperturbed equations we have two homoclinic solutions given by

$$
\gamma_{1}=\left(r_{1}, \dot{r}_{1}, 0,0\right), \quad \gamma_{2}=\left(0,0, r_{2}, \dot{r}_{2}\right)
$$

where $r_{1}(t)=\left(2 a_{1} / \sqrt{\pi}\right) \operatorname{sech} a_{1} t$ and $r_{2}(t)=\left(2 a_{2} / \sqrt{\pi}\right) \operatorname{sech} a_{2} t$.

Using $\gamma_{1}$ the adjoint equations (3.3) become

$$
\begin{aligned}
& \dot{v}_{1}=\left(-a_{1}^{2}+\frac{3 \pi}{2} r_{1}^{2}\right) v_{2} \\
& \dot{v}_{2}=-v_{1} \\
& \dot{v}_{3}=\left(-a_{2}^{2}+\frac{\pi}{2} r_{1}^{2}\right) v_{4} \\
& \dot{v}_{4}=-v_{3} .
\end{aligned}
$$

The essential question here is to determine the space of bounded solutions to these equations. We can write these in the form

$$
\ddot{v}_{2}=\left(a_{1}^{2}-\frac{3 \pi}{2} r_{1}^{2}\right) v_{2}, \quad \ddot{v}_{4}=\left(a_{2}^{2}-\frac{\pi}{2} r_{1}^{2}\right) v_{4} .
$$

The $v_{2}$ equation has a one-dimensional space of bounded solutions spanned by the solution $v_{2}=\dot{r}_{1}$, obtained from $\dot{\gamma}_{1}$. For the $v_{4}$ equation we have the following result.

LEMMA 2. Let $\kappa>0$. The equation

$$
\ddot{v}+\left(-\lambda+\kappa \operatorname{sech}^{2} t\right) v=0
$$

has a bounded solution if and only if there exists an integer $M$ such that

$$
\begin{array}{rlll}
\lambda & =\frac{1}{4}(\sqrt{4 \kappa+1}-4 M-1)^{2} & \text { for } & 0 \leq M<\frac{1}{4}(\sqrt{4 \kappa+1}-1) \\
\text { or } \quad \lambda & =\frac{1}{4}(\sqrt{4 \kappa+1}-4 M-3)^{2} & \text { for } & 0 \leq M<\frac{1}{4}(\sqrt{4 \kappa+1}-3) .
\end{array}
$$

The idea for the proof of this lemma is to express the solution as the product of a power of sech $t$ and a hypergeometric function with argument $-\sinh ^{2} t$. The condition for the existence of a bounded solution is that the hypergeometric series terminate and the resulting polynomial be of sufficiently small degree. The details for this have been worked out by Yagasaki in Appendix of [40]. See also Sections 23,25 of $[\mathbf{2 0}]$. 
Applying Lemma 2 to the equation for $v_{4}$ we find that the condition for a bounded solution is $a_{1}=a_{2}$ which is ruled out by the assumption $R>1$. Hence, the system of equations for $v$ has a one dimensional space of bounded solutions spanned by $v=\left(-\ddot{r}_{1}, \dot{r}, 0,0\right)$ and the Melnikov function (3.4) is

$$
M(\alpha)=\left[\frac{8 \omega_{0} \cos \zeta}{\sqrt{\pi}} \sin \omega_{0} \alpha \operatorname{sech} \frac{\pi \omega_{0}}{2 a_{1}}\right] \mu_{1}-\left(\frac{16 a_{1}^{3} \cos \eta}{3 \pi}\right) \mu_{2} .
$$

The non-resonance hypothesis follows as in the previous examples which leads, in the present case, to the following result obtained from Theorem 5 .

THEOREM 8. If $\omega_{0} \neq \omega_{n, i}$ for all $n$ and for $i=1,2$, then whenever $\mu_{0}$ satisfies $\mu_{0,1} \neq 0$ and

$$
0<\left|\frac{\mu_{0,2}}{\mu_{0,1}}\right|<\frac{3 \sqrt{\pi} \omega_{0} \cos \zeta}{2 a_{1}^{3} \cos \eta} \operatorname{sech} \frac{\pi \omega_{0}}{2 a_{1}}
$$

there exists a corresponding $\bar{\xi}_{0}>0$ such that if $0<\xi \leq \bar{\xi}_{0}$, if the parameters in (5.14) are given by $\mu=\xi \mu_{0}$ then there exists a compact subset of $\mathbb{R}^{4} \times \mathbb{Y}$ on which the 2 th iterate, $F^{2 m}$, of the period map $F$ of (5.14) is invariant and conjugate to the Bernoulli shift on $\Sigma$.

Replacing $\gamma_{1}$ with $\gamma_{2}$ yields the following analogous result.

THEOREM 9. If $\omega_{0} \neq \omega_{n, i}$ for all $n$ and for $i=1,2$, then whenever $\mu_{0}$ satisfies $\mu_{0,1} \neq 0$ and

$$
0<\left|\frac{\mu_{0,2}}{\mu_{0,1}}\right|<\frac{3 \sqrt{\pi} \omega_{0} \sin \zeta}{2 a_{2}^{3} \sin \eta} \operatorname{sech} \frac{\pi \omega_{0}}{2 a_{2}}
$$

there exists a corresponding $\bar{\xi}_{0}>0$ such that if $0<\xi \leq \bar{\xi}_{0}$, if the parameters in (5.14) are given by $\mu=\xi \mu_{0}$ then there exists a compact subset of $\mathbb{R}^{4} \times \mathbb{Y}$ on which the $2 m$ th iterate, $F^{2 m}$, of the period map $F$ of (5.14) is invariant and conjugate to the Bernoulli shift on $\Sigma$.

In the $\mu_{1}-\mu_{2}$ plane in this case we get a diagram as in Figure 3. For parameter values in the regions labeled $I$ there is one homoclinic orbit while for those in $I I$ there are two.

5.4. Multiple Buckled Modes. It remains to consider the situation where the axial load, $P_{0}$, is increased sufficiently to produce multiple buckled modes. We will look at the case of a beam constrained to planer motion. The calculations for the non-planer case are similar.

We return to the boundary value problem of Section 5.1 and use the same Galerkin equations

$$
\begin{aligned}
\ddot{u}_{n}=n^{2}\left(P_{0}-n^{2}\right) u_{n}-\frac{\pi}{2} n^{2}\left[\sum_{k=1}^{\infty} k^{2} u_{k}^{2}\right] u_{n} \\
\quad-2 \mu_{2} \dot{u}_{n}+2 \mu_{1}\left[\frac{1-(-1)^{n}}{\pi n}\right] \cos \omega_{0} t, \quad n=1,2, \ldots .
\end{aligned}
$$

In the present case we assume there exists an integer $N$ such that $N^{2}<P_{0}<$ $(N+1)^{2}$. We then define

$$
\begin{aligned}
a_{n}^{2} & =n^{2}\left(P_{0}-n^{2}\right), & & \text { for } n=1,2, \ldots, N ; \\
\omega_{n-N}^{2} & =n^{2}\left(n^{2}-P_{0}\right), & & \text { for } n=N+1, N+2, \ldots
\end{aligned}
$$


and put (5.15) in the form of (2.1) by defining

$$
\begin{aligned}
& x=\left(u_{1}, \dot{u}_{1}, u_{2}, \dot{u}_{2}, \ldots, u_{N}, \dot{u}_{N}\right), \\
& y=\left(u_{N+1}, \dot{u}_{N+1} / \omega_{1}, u_{N+2}, \dot{u}_{N+2} / \omega_{2}, \ldots\right) .
\end{aligned}
$$

A truncated version of the resulting equations with $N=2$ was studied in [40].

The reduced equations are

$$
\left.\begin{array}{rl}
\dot{x}_{2 n-1} & =x_{2 n} \\
\dot{x}_{2 n} & =a_{n}^{2} x_{2 n-1}-\frac{\pi n^{2}}{2}\left(\sum_{k=1}^{N} k^{2} x_{2 k-1}^{2}\right) x_{2 n-1} \\
& \quad-2 \mu_{2} x_{2 n}+2 \mu_{1}\left[\frac{1-(-1)^{n}}{\pi n}\right] \cos \omega_{0} t
\end{array}\right\} n=1,2, \ldots, N .
$$

When $\mu=0$ we have $N$ homoclinic solutions given by

$$
\gamma_{m}=(0, \ldots, 0, \underbrace{r_{m}, \dot{r}_{m}}_{2 m-1,2 m}, 0, \ldots, 0), \quad m=1,2, \ldots, N
$$

where $r_{m}(t)=\left(2 a_{m} / m^{2} \sqrt{\pi}\right) \operatorname{sech} a_{m} t$ and the adjoint equation (3.3) along $\gamma_{m}$ is

$$
\begin{aligned}
\dot{v}_{2 n-1} & =\left(-a_{n}^{2}+\frac{\pi m^{2} n^{2}}{2} r_{m}^{2}\right) v_{2 n}, \\
\dot{v}_{2 n} & =-v_{2 n-1}, \\
\dot{v}_{2 m-1} & =\left(-a_{m}^{2}+\frac{3 \pi m^{4}}{2} r_{m}^{2}\right) v_{2 m}, \\
\dot{v}_{2 m} & =-v_{2 m-1} .
\end{aligned}
$$

For the distinguished equation we have the bounded solution $v_{2 m-1}=-\ddot{r}_{m}, v_{2 m}=$ $\dot{r}_{m}$ while for the equations with $n \neq m$ we must solve

$$
\frac{d^{2} v_{2 n}}{d x^{2}}=\left(\frac{a_{n}^{2}}{a_{m}^{2}}-\frac{2 n^{2}}{m^{2}} \operatorname{sech}^{2} x\right) v_{2 n} .
$$

Using Lemma 2 we find that this last equation has a bounded solution if and only if there is an integer $M$ such that one of the following conditions hold:

$$
\begin{aligned}
& \frac{n^{2}\left(P_{0}-n^{2}\right)}{m^{2}\left(P_{0}-m^{2}\right)}= \frac{1}{4}\left[\sqrt{\frac{8 n^{2}}{m^{2}}+1}-4 M-1\right]^{2} \\
& \text { for } 0 \leq M<\frac{1}{4}\left(\sqrt{\frac{8 n^{2}}{m^{2}}+1}-1\right), \\
& \frac{n^{2}\left(P_{0}-n^{2}\right)}{m^{2}\left(P_{0}-m^{2}\right)}=\frac{1}{4}\left[\sqrt{\frac{8 n^{2}}{m^{2}}+1}-4 M-3\right]^{2} \\
& \quad \text { for } 0 \leq M<\frac{1}{4}\left(\sqrt{\frac{8 n^{2}}{m^{2}}+1}-3\right) .
\end{aligned}
$$

If, for some fixed $m$, none of the equations in (5.16) are satisfied for $n \neq m$ we can proceed much as in Section 5.1 since then the adjoint equation obtained from $\gamma_{m}$ has a one-dimensional space of bounded solutions spanned by

$$
v=(0, \ldots, 0, \underbrace{-\ddot{r}_{m}, \dot{r}_{m}}_{2 m-1,2 m}, 0, \ldots, 0) .
$$

One complication has been introduced by our assumption in the original partial differential equation that the transverse applied load is uniform in $x$. This assumption causes the $\mu_{1}$ terms to drop out in (5.15) for $n$ even which prohibits nonsingular 
solutions of $M(\alpha)=0$ as can be seen by examining Section 5.1. For this reason, we must choose $m$ odd. Theorem 5 now yields the following result.

THEOREM 10. Let $m$ be an odd integer, $1 \leq m \leq N$, and suppose $P_{0}$ is chosen so that none of the equations in (5.16) is satisfied. If $\omega_{0} \neq \omega_{n}$ for all $n$, then whenever $\mu_{0}$ satisfies $\mu_{0,1} \neq 0$ and

$$
0<\left|\frac{\mu_{0,2}}{\mu_{0,1}}\right|<\frac{3 m \sqrt{\pi} \omega_{0}}{2 a_{m}^{3}} \operatorname{sech} \frac{\pi \omega_{0}}{2 a_{m}}
$$

there exists a corresponding $\bar{\xi}_{0}>0$ such that if $0<\xi \leq \bar{\xi}_{0}$, if the parameters in (5.15) are given by $\mu=\xi \mu_{0}$ then there exists a compact subset of $\mathbb{R}^{2 N} \times \mathbb{Y}$ on which the $2 k$ th iterate, $F^{2 k}$, of the period map $F$ of (5.15) is invariant and conjugate to the Bernoulli shift on $\Sigma$.

We can simplify the preceding results by finding cases where the equations in (5.16) can never have a solution. The following is a helpful result along these lines.

LEMMA 3. The equations in (5.16) can never be satisfied for $n<m \leq N$.

Proof. For $(5.16 \mathrm{a})$ we have $\frac{1}{4}\left(\sqrt{8 n^{2} / m^{2}+1}-1\right)<\frac{1}{2}$ so we have only one equation to consider with $M=0$. But then we have, first, $\frac{n^{2}\left(P_{0}-n^{2}\right)}{m^{2}\left(P_{0}-m^{2}\right)}>\frac{n^{2}}{m^{2}}$, and also

$$
\frac{1}{4}\left[\sqrt{\frac{8 n^{2}}{m^{2}}+1}-1\right]^{2}-\frac{n^{2}}{m^{2}}=\frac{2 \frac{n^{2}}{m^{2}}\left(\frac{n^{2}}{m^{2}}-1\right)}{2 \frac{n^{2}}{m^{2}}+1+\sqrt{\frac{8 n^{2}}{m^{2}}+1}}<0
$$

so that the equation (5.16a) has no solution for any $P_{0}$.

Next we note that in when $n<m$, we have $\frac{1}{4}\left(\sqrt{8 n^{2} / m^{2}+1}-3\right)<0$ so that there are no equations for $(5.16 \mathrm{~b})$.

When $m=N$ the preceding result will eliminate any restriction, obtained from (5.16), on $P_{0}$. This fact was shown with a different technique by Berti and Carminati $[4]$ where they used a more general transverse forcing term which allowed for the possibility of a $\mu_{2}$ term for each $n$ in (5.15) and, hence, also for each $n$ in the reduced equation. They then take $m=N$. Since, for our specific form of loading, we must have $m$ odd we have the following result. hold:

THEOREM 11. Let $N$ and $P_{0}$ be as for (5.15) and suppose one of the following

(i) $N$ is odd and $m=N$.

(ii) $N$ is even, $N \geq 4, m=N-1$ and

$$
P_{0} \neq \frac{4 N^{2}-(N-1)^{2}\left[\sqrt{9 N^{2}-2 N+1}-3(N-1)\right]^{2}}{4 N^{2}-\left[\sqrt{9 N^{2}-2 N+1}-3(N-1)\right]^{2}} .
$$

(iii) $N=2, m=1$ and

$$
P_{0} \neq \frac{37+5 \sqrt{33}}{16}, \quad P_{0} \neq \frac{55+9 \sqrt{33}}{16} .
$$

Suppose in addition that $\omega_{n} \neq \omega_{0}$ for all $n$. Then whenever $\mu_{0}$ satisfies $\mu_{0,1} \neq 0$ and

$$
0<\left|\frac{\mu_{0,2}}{\mu_{0,1}}\right|<\frac{3 m \sqrt{\pi} \omega_{0}}{2 a_{m}^{3}} \operatorname{sech} \frac{\pi \omega_{0}}{2 a_{m}}
$$


there exists a corresponding $\bar{\xi}_{0}>0$ such that if $0<\xi \leq \bar{\xi}_{0}$, if the parameters in (5.15) are given by $\mu=\xi \mu_{0}$ then there exists a compact subset of $\mathbb{R}^{2 N} \times \mathbb{Y}$ on which the $2 k$ th iterate, $F^{2 k}$, of the period map $F$ of (5.15) is invariant and conjugate to the Bernoulli shift on $\Sigma$.

Proof. The result is obtained by using $\gamma_{m}$ and proceeding as in Section 5.1. This is valid as long as the equations (5.16) have no solutions for $n \neq m$ so it remains to show this is true in each case. If (i) holds we can use Lemma 3.

If $m=N-1$ then, using Lemma 3 , we need check only $n=N$. Define

$$
f_{a}(N)=\frac{1}{4}\left(\sqrt{\frac{8 N^{2}}{(N-1)^{2}}+1}-1\right), \quad f_{b}(N)=\frac{1}{4}\left(\sqrt{\frac{8 N^{2}}{(N-1)^{2}}+1}-3\right) .
$$

Then (5.16a) must be checked for integers $M \in\left[0, f_{a}(N)\right)$ and (5.16b) for integers $M \in\left[0, f_{b}(N)\right)$.

In case (ii) we have $N \geq 4$ which implies $1 / 2<f_{a}(N) \leq(\sqrt{137}-3) / 12<1$ so we need consider only $M=0$. In this case we solve

$$
\frac{N^{2}\left(P_{0}-N^{2}\right)}{(N-1)^{2}\left[P_{0}-(N-1)^{2}\right]}=4 f_{a}(N)^{2}
$$

for $P_{0}$ to get

$$
P_{0}=\frac{N^{4}-4 f_{a}(N)^{2}(N-1)^{4}}{N^{2}-4 f_{a}(N)^{2}(N-1)^{2}} .
$$

But this value is negative and so can be discarded.

Similarly, we have for $N \geq 4,0<f_{b}(N) \leq(\sqrt{137}-9) / 12<1$ so in $(5.16 \mathrm{~b})$ we need also consider only $M=0$. Here we get

$$
\begin{aligned}
P_{0} & =\frac{N^{4}-4 f_{b}(N)^{2}(N-1)^{4}}{N^{2}-4 f_{b}(N)^{2}(N-1)^{2}} \\
& =\frac{4 N^{4}-(N-1)^{2}\left[\sqrt{9 N^{2}-2 N+1}-3(N-1)\right]^{2}}{4 N^{2}-\left[\sqrt{9 N^{2}-2 N+1}-3(N-1)\right]^{2}} .
\end{aligned}
$$

Next, we consider (iii) where $N=2, m=1$. Since $2>f_{a}(2)=(\sqrt{33}-1) / 4>1$ we must consider $M=0$ and $M=1$ in (5.16a). When $M=0$ we get the value $P_{0}=-(7+\sqrt{33}) / 2<0$ which can be discarded while for $M=1$ we have $P_{0}=$ $(37+5 \sqrt{33}) / 16$.

Finally, $0<f_{b}(2)=(\sqrt{33}-3) / 4<1$ so only $M=0$ must be considered in (5.16b) and this yields $P_{0}=(55+9 \sqrt{33}) / 16$.

\section{Appendix A. Appendix}

We propose here a method for computing the constant $m_{2}$ of Section 5.2. For this reason, we first search for critical points of the function $\Phi(\beta)$ from equation (5.9). Note

$$
\Phi(\beta)=\frac{3 \omega_{0} \sqrt{\pi}}{2 a^{3}} \operatorname{sech} \frac{\pi \omega_{0}}{2 a} \Psi(\beta)
$$

for

$$
\Psi(\beta)=\frac{\cos (\beta-\zeta)}{\cos \eta \cos ^{2} \beta+\sin \eta \sin ^{2} \beta} .
$$


Since $\Psi(\beta+\pi)=-\Psi(\beta)$ and $\Psi(0)=\frac{\cos \zeta}{\cos \eta}>0, \Psi^{\prime}(0)=\frac{\sin \zeta}{\cos \eta}>0$, we restrict our analysis to $\beta \in(0, \pi)$. Taking $x:=\tan \beta / 2$ and using the formulas

$$
\sin \beta=\frac{2 \tan (\beta / 2)}{1+\tan ^{2}(\beta / 2)}=\frac{2 x}{1+x^{2}}, \quad \cos \beta=\frac{1-\tan ^{2}(\beta / 2)}{1+\tan ^{2}(\beta / 2)}=\frac{1-x^{2}}{1+x^{2}},
$$

we get $\Psi(\beta)=\Upsilon(x)$ for

$$
\Upsilon(x):=\frac{\left(1-x^{4}\right) \cos \zeta+2 x\left(1+x^{2}\right) \sin \zeta}{\left(x^{2}-1\right)^{2} \cos \eta+4 x^{2} \sin \eta} .
$$

We compute

$$
\Upsilon^{\prime}(x)=-\frac{2 f(x) \sin \eta \sin \zeta}{\left(\left(x^{2}-1\right)^{2} \cos \eta+4 x^{2} \sin \eta\right)^{2}}
$$

for

$$
\begin{aligned}
& f(x):=G x^{6}+x^{5}(4 Z-2 G Z)+x^{4}(5 G-4) \\
& +4 G Z x^{3}+x^{2}(4-5 G)+x(4 Z-2 G Z)-G,
\end{aligned}
$$

where $Z=\cot \zeta>0$ and $G=\cot \eta>0$. Hence $\Upsilon^{\prime}(x)=0, x>0$ if and only if

$$
f(x)=0 .
$$

Verifying

$$
x^{6} f(-1 / x)=-f(x) \quad \forall x \neq 0,
$$

$f(x)$ is an anti-reciprocal polynomial [33]. Due to $f(0)=-G<0$, certainly $f(x)=0$ has a positive root. Also if $f\left(x_{0}\right)=0$ then $f\left(-1 / x_{0}\right)=0$. Using (A.3), we put

$$
y:=x-x^{-1} .
$$

Note the mapping $x \mapsto x-x^{-1}$ is increasing on $(0, \infty)$ with the range $\mathbb{R}$. Its inverse is $x=\frac{y+\sqrt{y^{2}+4}}{2}$. Next we rewrite (A.2) as

$$
G x^{3}-G x^{-3}+\left(x^{2}+x^{-2}\right)(4 Z-2 G Z)+\left(x-x^{-1}\right)(5 G-4)+4 G Z=0 .
$$

From

$$
y^{2}=x^{2}+x^{-2}-2, \quad y^{3}=x^{3}-x^{-3}-3\left(x-x^{-1}\right),
$$

in the variable $y$, (A.4) has the form

$$
p(y):=G y^{3}+2 Z(2-G) y^{2}+4(2 G-1) y+8 Z=0 .
$$

Rewriting (A.1) in the variable $y$ we get

$$
\Theta(y)=\frac{2 \sin \zeta-y \cos \zeta}{y^{2} \cos \eta+4 \sin \eta} \sqrt{y^{2}+4} .
$$

Recall $y=x-x^{-1}, y=-2 \cot \beta$ and $\Psi(\beta)=\Upsilon(x)=\Theta(y)$ for $\beta \in(0, \pi), x \in(0, \infty)$, $y \in \mathbb{R}$. We can easily check that

$$
\Theta^{\prime}(y)=-\frac{2 p(y) \sin \zeta \sin \eta}{\sqrt{y^{2}+4}\left(y^{2} \cos \eta+4 \sin \eta\right)^{2}} .
$$

Hence $\Theta^{\prime}(y)=0$ if and only if $p(y)=0$. Note

$$
f(x)=x^{3} p\left(x-x^{-1}\right) .
$$

Summarizing, in order to find $m_{2}$, we need to solve (A.5) and then insert its solutions to (A.6). 


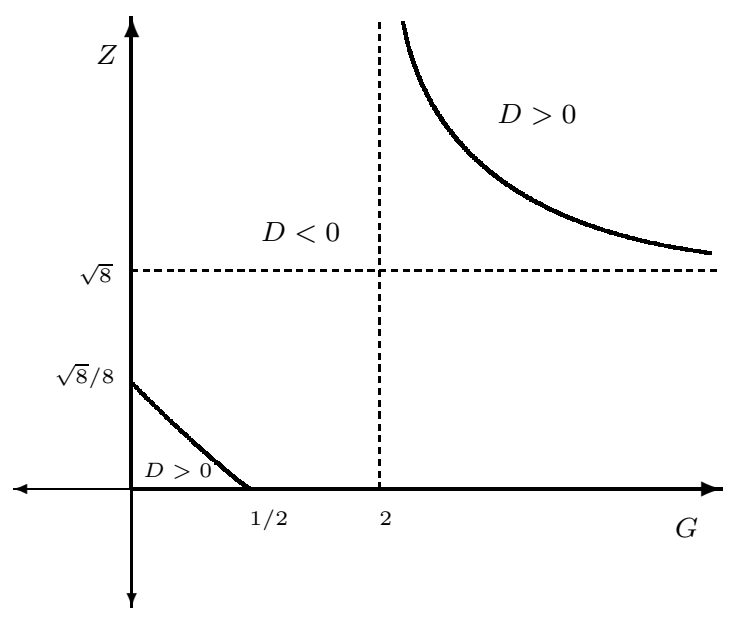

FiguRE 4. Sign regions of the discriminant $D$.

Next we compute the discriminant $D$ of (A.5) (see [5]):

$$
D=\frac{64}{27 G^{4}}\left(Z^{2}+G\right)\left((G-2)^{3} Z^{2}-(2 G-1)^{3}\right) .
$$

Now (see Figure 4) we have the following possibilities:

1) $D>0 \Leftrightarrow(G-2)^{3} Z^{2}>(2 G-1)^{3}$, and then (A.5) has 3 different real roots.

2) $D=0 \Leftrightarrow(G-2)^{3} Z^{2}=(2 G-1)^{3}$, and then (A.5) has 3 real roots, but one is double.

3) $D<0 \Leftrightarrow(G-2)^{3} Z^{2}<(2 G-1)^{3}$, and then (A.5) has 1 real root.

These roots are done by the Cardano formulas [5]. For general $\zeta$ and $\eta$ these formulas are rather awkward, but for concrete values of $\zeta$ and $\eta$, we can easily check which one of the above cases (1)-(3) hold, and then we easily compute the roots by using these Cardano formulas. Inserting these roots into (A.6) we are able to find $m_{2}$ for concrete values of $\zeta$ and $\eta$. Moreover, in the cases (2), (3) there is a unique simple zero $y_{0}$ of $p(y)$ and then $m_{2}=\frac{3 \omega_{0} \sqrt{\pi}}{2 a^{3}} \operatorname{sech} \frac{\pi \omega_{0}}{2 a} \Theta\left(y_{0}\right)$.

Summarizing, we suggest this simple algorithm/method for finding $m_{2}$ : For general $\zeta$ and $\eta$, the formula for $m_{2}$ is very awkward, so we do not derive it, but for concrete values of $\zeta$ and $\eta$, we can directly calculate $m_{2}$ avoiding the use of some numerical/approximation methods.

\section{References}

[1] Battelli, F. And FečKan, M. Chaos in the beam equation, J. Differential Equations 209 (2005), 172-227.

[2] Battelli, F., FeČKan, M. and Franca, M. On the chaotic behavior of a compressed beam, Dynamics of PDE 4 (2007), 55-86.

[3] Battelli, F. And Lazzari, C. Exponential dichotomies, heteroclinic orbits, and Melnikov functions, J. Differential Equations 86 (1990), 342-366.

[4] Berti, M and Carminati, C. Chaotic dynamics for perturbations of infinite dimensional Hamiltonian systems, Nonlinear Analysis 48 (2002), 481-504.

[5] Birkhoff, G. And Mac Lane, S. A Survey of Modern Algebra, 5th ed. Macmillan, New York, 1996. 
[6] Chen, G., Hsu, S-B. and Zhou, J. Chaotic vibration of the wave equation with nonlinear feedback boundary control: progress and open questions, in: G. Chen, X. Yu (Eds.), Chaos Control - Theory and Applications, Lecture Notes in Control and Information Sciences (LNCIS) 292, Berlin: Springer 2003, 25-50.

[7] Coppel, W. A. Dichotomies in Stability Theory, Lecture Notes in Mathematics, v. 629, Springer-Verlag, 1978.

[8] Devaney, R.: An Introduction to Chaotic Dynamical Systems, Benjamin/Cummings, Menlo Park, CA, 1989.

[9] FeČKan, M. Higher dimensional Melnikov mappings, Math. Slovaca 49 (1999), 75-83.

[10] Bifurcation of periodics and subharmonics in abstract nonlinear undamped wave equations, J. Differential Equations 153 (1999), 41-60.

[11] Chaos in non-autonomous differential inclusions, Int. J. Bif. Chaos 15 (2005), 19191930.

[12] FeČKAn, M. And Gruendler, J. The existence of chaos for ordinary differential equations with a center manifold, Bull. Belgian Math. Soc. 11 (2004), 77-94.

[13] Gruendler, J. Homoclinic solutions for autonomous dynamical systems in arbitrary dimension, SIAM J. Math. Anal. 23(3) (1992), 702-721.

[14] - Homoclinic solutions for autonomous ordinary differential equations with nonautonomous perturbations, J. Differential Equations 122(1) (1995), 1-26.

[15] The existence of transverse homoclinic solutions for higher order equations, J. Differential Equations 130(2) (1996), 307-320.

[16] Hale, J. K. Ordinary Differential Equations, Robert E. Krieger, New York, 2nd ed., 1980.

[17] Holmes, P. A nonlinear oscillator with a strange attractor, Phil. Trans. Roy. Soc. A 292 (1979), 419-448.

[18] Holmes, P. And Marsden, J. A partial differential equation with infinitely many periodic orbits: chaotic oscillations of a forced beam, Arch. Rational Mech. Anal. 76 (1981), 135-165.

[19] Irwin, M. C. Smooth Dynamical Systems, Academic Press, New York, 1980.

[20] Landau, L. D. and Lifshitz, E. M. Quantum Mechanics (Non-relativistic Theory), Elsevier, New York, 3rd ed., 1994.

[21] LI, Y. Persistent homoclinic orbits for nonlinear Schrödinger equation under singular perturbation, Dynamics of PDE 1 (2004), 87-123.

[22] - Smale horseshoes and symbolic dynamics in perturbed nonlinear Schrödinger equations, Journal of Nonlinear Sciences 9 (1999), 363-415.

[23] — Existence of chaos for nonlinear Schrödinger equation under singular perturbation, Dynamics of PDE 1 (2004), 225-237.

[24] Chaos and shadowing lemma for autonomous systems of infinite dimensions, J. Dyn. Diff. Eq. 15 (2003), 699-730.

[25] Homoclinic tubes and chaos in perturbed sine-Gordon equation, Chaos, Solitons and Fractals 20 (2004), 791-798.

[26] Chaos and shadowing around a heteroclinically tubular cycle with an application to sine-Gordon Equation, Studies in Applied Mathematics 116 (2006), 145-171.

[27] Li, Y. And McLaughlin, D. Morse and Melnikov functions for NLS Pde's., Comm. Math. Phys. 162 (1994), 175-214.

[28] Li, Y., Mclaughlin, D., Shatah, J. and Wiggins, S. Persistent Homoclinic Orbits for Perturbed Nonlinear Schrödinger Equation, Comm. Pure and Appl. Math. 49 (1996), 11751255.

[29] Palmer, K. J. Exponential dichotomies and transversal homoclinic points, J. Differential Equations 55 (1984), 225-256.

[30] Shadowing in Dynamical Systems, Kluwer Academic Publishers, Dordrecht, 2000.

[31] Robinson, C. Dynamical Systems. Stability, Symbolic Dynamics, and Chaos, CRC Press, Boca Raton, 1995.

[32] Rodrigues, H. M. and Silveira, M. Properties of bounded solutions of linear and nonlinear evolution equations: homoclinics of a beam equation, J. Differential Equations 70 (1987), 403-440.

[33] Sinclair, CH. D. The distribution of Mahler's measures of reciprocal polynomials, International Journal of Mathematics and Mathematical Sciences 2004 (2004), 2773-2786.

[34] Smale, S. Differentiable dynamical systems, Bull. Amer. Math. Soc. 73 (1967), 747-817. 
[35] Wiggins, S. Global Bifurcations and Chaos, Analytical Methods, Applied Mathematical Sciences 73, Springer-Verlag, New York, 1988.

[36] ZeLIK, S.V. The attractor for a nonlinear reaction-diffusion system in the unbounded domain and Kolmogorov's $\epsilon$-entropy, Math. Nachr. 232 (2001), 129-179.

[37] The attractor for a nonlinear hyperbolic equation in the unbounded domain, Disc. Cont. Dyn. Sys. Ser. A 7 (2001), 593-641.

[38] - Attractors of reaction-diffusion systems in unbounded domains and their spatial complexity, Comm. Pure Appl. Math. 56 (2003), 584-637.

[39] ZHAO, Y. Introduction to some methods of chaos analysis and control for PDEs, in: $G$. Chen, X. Yu (Eds.), Chaos Control - Theory and Applications, Lecture Notes in Control and Information Sciences (LNCIS) 292, Berlin: Springer 2003, 89-115.

[40] YAGASAKI, K. The method of Melnikov for perturbations of multi-degree-of-freedom Hamiltonian systems, Nonlinearity 12 (1999), 799-822.

[41] Homoclinic and heteroclinic behavior in an infinite-degree-of-freedom Hamiltonian system: chaotic free vibrations of an undamped, buckled beam, Phys. Lett. A 285 (2001), 55-62.

Department of Mathematical Analysis and Numerical Mathematics, Comenius UniVERSity, Mlynská dolina, 84248 BratislaVa - Slovakia, and Mathematical Institute, Slovak Academy of Sciences, Štefánikova 49, 81473 Bratislava - Slovakia

E-mail address: Michal.Feckan@fmph.uniba.sk

37 Fountain Manor Drive, Suite B, Greensboro, North Carolina 27405-8077, USA 